\title{
La cultura como estrategia de regeneración urbana en Monterrey, México
}

\author{
Fabiola R. Garza-Rodríguez \\ Nancy Andrea Ramírez-Agudelo \\ Elisabet Roca Bosch \\ Míriam Villares Junyent
}

Universitat Politècnica de Catalunya. Institut universitari de recerca en Ciència i Tecnologies de la Sostenibilitat

fgarzar@uanl.edu.mx

nancy.andrea.ramirez@upc.edu

elisabet.roca@upc.edu

miriam.villares@upc.edu

\section{Resumen}

La cultura se ha utilizado como estrategia de regeneración urbana por su permeabilidad hacia otras áreas del desarrollo. Desde hace más de 30 años, el centro de Monterrey ha experimentado deterioro, pérdida de población y abandono, con lo que la estrategia del sector público ha sido realizar proyectos de regeneración urbana con fuerte implicación cultural. A pesar de la magnitud y la inversión de los proyectos, no se han logrado los objetivos esperados. En cambio, a partir del año 2013 surgen proyectos desde iniciativas bottom-up que están transformando positivamente el lugar. Se analizan los proyectos de regeneración urbana implementados en el centro mediante entrevistas a actores clave. Entre los principales resultados, destacan las iniciativas bottom-up como procesos de regeneración cultural capaces de transformar el territorio y el tejido social. La falta de diálogo entre actores es uno de los principales obstáculos para producir transformaciones más contundentes en el contexto sociourbano.

Palabras clave: regeneración urbana; políticas; centro de Monterrey; regeneración cultural 
Resum. La cultura com a estratègia de regeneració urbana a Monterrey, Mèxic

La cultura s'ha utilitzat com a estratègia de regeneració urbana per la seva permeabilitat cap a altres àrees del desenvolupament. Des de fa més de 30 anys, el centre de Monterrey ha experimentat deteriorament, pèrdua de població i abandonament, de manera que l'estratègia del sector públic ha estat realitzar projectes de regeneració urbana amb forta implicació cultural. Tot i la magnitud i la inversió dels projectes, no s'han aconseguit els objectius esperats. En canvi, a partir de l'any 2013 sorgeixen projectes des d'iniciatives bottom-up que estan transformant positivament el lloc. S'analitzen els projectes de regeneració urbana mitjançant entrevistes a actors clau. Entre els principals resultats, destaquen les iniciatives bottom-up com a processos de regeneració cultural capaços de transformar el territori i el teixit social. La falta de diàleg entre actors és un dels principals obstacles per produir transformacions més contundents en el context sociourbà.

Paraules clau: regeneració urbana; polítiques; Monterrey centre; regeneració cultural

Résumé. La culture en tant que stratégie de revitalisation urbaine à Monterrey, au Mexique

La culture a été utilisée comme stratégie de régénération urbaine en raison de sa perméabilité à d'autres domaines de développement. Pendant plus de 30 ans, le centre de Monterrey a connu une détérioration, une perte de population et un abandon, alors que le secteur public avait pour stratégie de mener à bien des projets de revitalisation urbaine à forte implication culturelle. Malgré l'ampleur et l'investissement des projets, ceux-ci n'ont pas atteint les objectifs escomptés. D'autre part, depuis 2013, les projets sont issus d'initiatives ascendantes qui transforment positivement le lieu. Les projets de régénération sont analysés à travers des entretiens avec des acteurs clés. Parmi les principaux résultats, citons les initiatives ascendantes en tant que processus de régénération culturelle capables de transformer le territoire et le tissu social. L'absence de dialogue entre les acteurs est l'un des principaux obstacles à une transformation plus forte du contexte socio-urbain.

Mots-clés: régénération urbaine; politiques; Monterrey centre-ville; régénération culturelle

\section{Abstract. Culture as an urban regeneration strategy in Monterrey, Mexico}

Culture has been used as an urban regeneration strategy due to its permeability to other areas of development. For more than 30 years, the center of Monterrey, Mexico, has experienced deterioration, population loss and abandonment, where the strategy of the public sector has been to carry out urban regeneration projects with strong cultural involvement. Despite the magnitude and investment of these projects, they have not achieved the expected objectives. Since 2013, however, projects have emerged from bottom-up initiatives that are positively transforming the place. Through interviews with key actors, this paper analyzes urban regeneration projects implemented in the center of Monterrey. Among the main results, cultural regeneration processes based on bottom-up initiatives are revealed to be capable of transforming the territory and the social fabric. However, the lack of dialogue between actors is one of the main obstacles to produce more forceful transformations in the socio-urban context.

Keywords: urban regeneration; politics; Monterrey center; cultural regeneration 


\section{Sumario}

1. Introducción

2. Sostenibilidad y cultura como herramientas de planeamiento urbanístico

3. Caso de estudio: Área Metropolitana de Monterrey (AMM) y su centro metropolitano
4. Narrativas a partir de las intervenciones urbanísticas en torno al centro

5. Discusión: hacia regeneraciones urbanas colaborativas

6. Conclusión

Referencias bibliográficas

\section{Introducción}

Las regeneraciones urbanas impulsadas por la cultura han sido ampliamente investigadas en contextos norteamericanos, europeos (García, 2004; Jones y Evans, 2008) y asiáticos (Kana, 2012; Jung et al., 2015). Aunque en el contexto latinoamericano existen estudios que analizan este proceso (Yúdice, 2008; Kanai y Ortega-Alcázar, 2009; Becker y Muller, 2013; Duque Franco, 2015; RiusUlldemolins y Posso Jiménez, 2016), siendo los casos más comunes Medellín y Bogotá (Duque Franco, 2015), hace falta extrapolar la investigación hacia otros contextos, como el mexicano. Con el objetivo de entender estas regeneraciones, cómo se producen y surgen narrativas a partir de las intervenciones urbanísticas, se dispone de un campo de investigación que puede explorar su impacto.

El área metropolitana de Monterrey (AMM) concentra una población de más de cuatro millones de habitantes en 2018. Su consolidación como enclave logístico, industrial y de negocios se ve favorecida por su localización geográfica. Desde los años ochenta, el gobierno local y estatal apuestan por la regeneración urbana del centro a través de proyectos emblemáticos y megaeventos. A pesar de la intención de posicionar la ciudad internacionalmente, el centro continúa luchando contra fenómenos como pérdida de población, el peso del sector terciario, el deterioro material y el abandono (García Ortega, 2001). El hecho de que el AMM continúe creciendo y desarrollándose y que, por otro lado, el centro se encuentre ante estas problemáticas muestra de realidades alternas en la misma ciudad.

Las problemáticas generalizadas en los centros de ciudades latinoamericanas, como la degradación, el abandono y la pérdida de población, son consideradas áreas de oportunidad para fomentar su revitalización (García et al., 2016). En ese sentido, la cultura se convierte en una herramienta estratégica en la política urbana (Dinardi, 2012), para fomentar su potencial urbano, cultural e histórico. Este trabajo analiza las transformaciones urbanas realizadas en los últimos 30 años en el centro de la ciudad de Monterrey, partiendo de la hipótesis de que el análisis de los proyectos de regeneración urbana vistos desde las narrativas de actores clave facilita el entendimiento del modelo de gobernanza y las estrategias implementadas. Por lo tanto, ya que este artículo busca avanzar en los argumentos sobre cómo las estrategias podrían considerarse mecanismos de sostenibilidad cultural, se proponen los siguientes objetivos: 
Analizar las transformaciones urbanas llevadas a cabo en el centro de Monterrey desde 1980 hasta la fecha partiendo de sistemas de gobernanza y de criterios urbanísticos asociados a la regeneración cultural.

Identificar las narrativas a partir de la gestión urbanística, mediante entrevistas a actores clave para crear un espacio temporal sobre el centro de la ciudad de Monterrey, con la finalidad de evidenciar percepciones sociales del centro.

Visibilizar estrategias que pueden asociarse al término de sostenibilidad cultural en la regeneración urbana de un centro latinoamericano.

El análisis presenta lecciones aprendidas e incorpora argumentos para promover mecanismos de sostenibilidad cultural, como la gestión colaborativa en las intervenciones de regeneración urbana para facilitar el diálogo entre actores (académicos, cívicos) y para garantizar continuidad en la gestión pública y el interés económico, con retorno social de los actores público-privados.

\section{Sostenibilidad y cultura como herramientas de planeamiento urbanístico}

La regeneración urbana es un proceso sistémico que parte de una acción gubernamental, de regulaciones y planeamiento para transformar un lugar con deterioro físico, social, ambiental, económico (Evans, 2005; Roberts et al., 2016) y cultural. Hoy en día, la regeneración urbana busca objetivos relacionados con el bienestar social y ambiental, para crear ciudades compactas, saludables, equitativas, eficientes, creativas y cohesionadas (Evans, 2005; Florida, 2005). De esta forma, promueve factores cualitativos y de bienestar que influyen en la calidad de vida y en la resiliencia urbana (Heath et al., 2017).

El aporte de la cultura en la ciudad se manifiesta a través de la creatividad, en medios de expresión artística, en el desarrollo de identidad colectiva (Zukin, 1987; Harvey, 1989; Bianchini y Parkinson, 1993; Landry, 2003), en la creación de macroeventos y proyectos emblemáticos (García, 2004; Shin, 2010; Tallon, 2013; Jung et al., 2015), en la implementación de políticas públicas culturales (Bianchini y Parkinson, 1993; Landry y Bianchini, 1995) y de gobernanza urbana (Degen y García, 2012). Cuando las estrategias culturales, más allá de crecimiento económico, generan mejores formas de vida, se produce desarrollo urbano y social. De esta manera contribuyen al discurso de sostenibilidad urbana, ya que, al mejorar la forma y el funcionamiento de la urbe, con el fortalecimiento de diálogos entre actores y el refuerzo de los valores que caracterizan y moldean a una sociedad, se mejora la experiencia de vivir en ella (Landry y Bianchini, 1995; Pethia, 2011).

Las regeneraciones urbanas se producen desde diversos modelos de gobernanza. El modelo top-down consiste en la coordinación de inversión del capital - ya sea público o privado- ajustándose a un plan de reforma urbana, como el vinculado al distrito 22@ en Barcelona (García et al., 2016). El modelo bottom-up surge a partir de iniciativas de residentes locales que producen, crean y gestionan lo relativo a la intervención, como sucede en el barrio de Savamala, 
Tabla 1. Caracterización de la cultura a partir de la revisión de literatura

\begin{tabular}{|c|c|c|}
\hline Caracterización de la cultura & Descripción & Autores \\
\hline \multirow[t]{3}{*}{ Agenda pública } & $\begin{array}{l}\text { En la transformación urbana como: } \\
\text { - Instrumento } \\
\text { - Herramienta }\end{array}$ & $\begin{array}{l}\text { Zukin, 1995; } \\
\text { Bayliss, 2004; } \\
\text { UN-Habitat, 2004; } \\
\text { Miles, 2005; } \\
\text { Pratt, } 2009\end{array}$ \\
\hline & $\begin{array}{l}\text { En la implementación de políticas } \\
\text { públicas culturales }\end{array}$ & $\begin{array}{l}\text { Bianchini y Parkinson, 1993; } \\
\text { Landry y Bianchini, } 1995\end{array}$ \\
\hline & $\begin{array}{l}\text { En la implementación } \\
\text { de gobernanza urbana }\end{array}$ & Degen y García, 2012 \\
\hline \multirow[t]{2}{*}{ Manifestación } & $\begin{array}{l}\text { Manifestación del aporte de la } \\
\text { cultura en estrategias enfocadas } \\
\text { en: } \\
\text { - La creatividad } \\
\text { - Medios de expresión } \\
\text { artística } \\
\text { - El desarrollo de identidad } \\
\text { colectiva }\end{array}$ & $\begin{array}{l}\text { Zukin, 1987; } \\
\text { Harvey, 1989; } \\
\text { Bianchini y Parkinson, 1993; } \\
\text { Landry, } 2003\end{array}$ \\
\hline & $\begin{array}{l}\text { Manifestación en la creación de: } \\
\text { - Macroeventos } \\
\text { - Proyectos emblemáticos }\end{array}$ & $\begin{array}{l}\text { García, 2004; } \\
\text { Shin, 2010; } \\
\text { Tallon, 2013; } \\
\text { Jung et al., } 2015 \\
\text { Evans, 2005 } \\
\text { Montgomery, } 2003\end{array}$ \\
\hline $\begin{array}{l}\text { Método alternativo de } \\
\text { desarrollo urbano y social }\end{array}$ & $\begin{array}{l}\text { Generador de mejores formas } \\
\text { de vida en la ciudad. Método } \\
\text { alternativo de desarrollo urbano } \\
\text { y social: } \\
\text { - Contribuyendo a la sostenibili- } \\
\text { dad urbana, no solo por mejo- } \\
\text { rar la forma y el funcionamiento } \\
\text { de la urbe, sino por mejorar la } \\
\text { experiencia de vivir en ella } \\
\text { - Fortaleciendo diálogos entre } \\
\text { actores } \\
\text { - Reforzando así los valores que } \\
\text { caracterizan y moldean a una } \\
\text { sociedad }\end{array}$ & $\begin{array}{l}\text { Landry y Bianchini, 1995; } \\
\text { Pethia, } 2011\end{array}$ \\
\hline
\end{tabular}

Fuente: elaboración propia.

en Belgrado, Serbia (Lazarevič et al., 2016). Además, están las estrategias culturales que surgen a partir de asociaciones entre actores (público, privado, civil), que permiten gobernanzas más colaborativas y transversales. Un buen ejemplo es la restauración de una fábrica harinera en Zaragoza como equipamiento cultural autogestionado (Harinera ZGZ), cuya clave de funcionamiento y consolidación ha sido a partir de un modelo de gobernanza colaborativo entre el sector público y el civil (López Senabre y Holst, 2017). 
Tabla 2. Resumen de la gobernanza en la regeneración urbana y estrategias culturales

\begin{tabular}{lll}
\hline $\begin{array}{l}\text { Caracterización } \\
\text { de la gobernanza }\end{array}$ & Descripción & Casos - Ejemplos \\
\hline Top-down & $\begin{array}{l}\text { Coordinación de inversión del capital - público } \\
\text { o privado - ajustándose a un plan maestro o de } \\
\text { reforma urbana }\end{array}$ & $\begin{array}{l}\text { Distrito 22@ en } \\
\text { Barcelona }\end{array}$ \\
\hline Bottom-up & $\begin{array}{l}\text { Residentes locales producen, crean y gestionan } \\
\text { lo relativo al proyecto }\end{array}$ & $\begin{array}{l}\text { Barrio de Savamala, } \\
\text { en Belgrado (Serbia) }\end{array}$ \\
\hline $\begin{array}{l}\text { Asociaciones } \\
\text { entre actores }\end{array}$ & Permiten trabajos más colaborativos (público, & $\begin{array}{l}\text { Restauración de } \\
\text { una fábrica harinera } \\
\text { privado, civil) y transversales } \\
\text { equipamiento cultural } \\
\text { autogestionado } \\
\text { (Harinera ZGZ) }\end{array}$ \\
\hline
\end{tabular}

Fuente: elaboración propia.

Tabla 3. Caracterización de los tipos de estrategias culturales a partir de la literatura

\begin{tabular}{|c|c|c|c|c|c|}
\hline $\begin{array}{l}\text { Estrategias } \\
\text { culturales }\end{array}$ & Gobernanza & Modelo de gestión & Descripción & Objetivo & Casos - Ejemplos \\
\hline \multirow{3}{*}{$\begin{array}{l}\text { Renovación } \\
\text { urbana }\end{array}$} & \multirow[t]{3}{*}{ Top-down } & \multirow{3}{*}{$\begin{array}{l}\text { Asociaciones } \\
\text { público-privadas }\end{array}$} & Planes & \multirow{3}{*}{$\begin{array}{l}\text { Revitalizar un área } \\
\text { urbana con la presencia } \\
\text { de un ejemplar arqui- } \\
\text { tectónico de marca (o } \\
\text { de un star-architect) }\end{array}$} & \multirow{3}{*}{$\begin{array}{l}\text { Guggenheim en Bilbao } \\
\text { MACBA en Barcelona } \\
\text { Plan CHA en Paraguay }\end{array}$} \\
\hline & & & $\begin{array}{l}\text { Proyectos emble- } \\
\text { máticos }\end{array}$ & & \\
\hline & & & $\begin{array}{l}\text { Equipamientos } \\
\text { culturales }\end{array}$ & & \\
\hline \multirow[t]{2}{*}{$\begin{array}{l}\text { Industrias } \\
\text { culturales } \\
0 \text { creativas }\end{array}$} & Top-down & Política pública & Distritos culturales & $\begin{array}{l}\text { Generar distritos cul- } \\
\text { turales a partir de la } \\
\text { concentración de acti- } \\
\text { vidades relacionadas } \\
\text { con la industria cultural, } \\
\text { creativa y artística }\end{array}$ & $\begin{array}{l}\text { Concentración de } \\
\text { artistas en el sector del } \\
\text { Poblenou, Barcelona } \\
\text { (años 90) }\end{array}$ \\
\hline & Bottom-up & $\begin{array}{l}\text { Iniciativa } \\
\text { de residentes }\end{array}$ & & & $\begin{array}{l}\text { A partir del movimiento } \\
\text { chicano en el Barrio } \\
\text { Logan, en San Diego } \\
\text { (Estados Unidos) }\end{array}$ \\
\hline \multirow{5}{*}{$\begin{array}{l}\text { Políticas } \\
\text { públicas } \\
\text { culturales }\end{array}$} & \multirow[t]{5}{*}{ Top-down } & \multirow[t]{5}{*}{ Pública } & Agendas culturales & Incluir agendas cultura- & \multirow{5}{*}{$\begin{array}{l}\text { Plan Maestro de Equi- } \\
\text { pamientos Culturales } \\
\text { (Plamec) en Bogotá } \\
\text { (Colombia) }\end{array}$} \\
\hline & & & Eventos & les apoyando procesos & \\
\hline & & & Exposiciones & & \\
\hline & & & Conciertos & & \\
\hline & & & Festivales & & \\
\hline
\end{tabular}

Fuente: elaboración propia.

Los procesos de regeneración cultural ofrecen un enfoque alternativo al análisis de la práctica urbana (Montgomery, 2004; García, 2004; Evans, 2005; Miles, 2005; Degen y García, 2012; Rius-Ulldemolins y Posso Jiménez, 2016). En este caso, el análisis cualitativo reconoce como fuente de información a 
Tabla 4. Descripción de elementos de la narrativa

\begin{tabular}{llll}
\hline Elementos & El juicio & El proyecto & El programa \\
\hline Descripción & El entrevistado hace & Lo que podría cambiarse & Cómo podrían lograrse \\
& juicios de valor sobre: & (para regenerar el centro) & estos objetivos: \\
& - Las problemáticas & Lo que está sucediendo & $\bullet$ Identificación de \\
& del centro & actualmente (para & áreas de oportunidad \\
& - Quién las produce & regenerar el centro) & - Identificación de \\
& $\bullet$ Qué las produce & & estrategias puntuales
\end{tabular}

Fuente: elaboración propia a partir de Mercier (2008).

Tabla 5. Descripción de herramientas metodológicas

\begin{tabular}{l} 
Revisión documental \\
\hline Descripción \\
- Acceso a fuentes primarias \\
- Revisión de trabajos académicos \\
- Documentos históricos \\
- Planes urbanísticos locales
\end{tabular}

\section{Entrevistas}

Descripción

- 15 entrevistas semiestructuradas

- Presenciales

- Obtenidas durante el periodo 2015-2018

- De 45' a 120' de duración

- Grabadas y transcritas

- Análisis posterior

\begin{tabular}{|c|c|}
\hline $\begin{array}{l}\text { Fuente de información } \\
\text { de los expertos }\end{array}$ & $\begin{array}{l}\text { Cuatro actores expertos } \\
\text { - Académicos / investiga- } \\
\text { dores en arquitectura } \\
\text { - Urbanismo } \\
\text { - Conservación del } \\
\text { patrimonio }\end{array}$ \\
\hline $\begin{array}{l}\text { Fuente de información } \\
\text { oficial }\end{array}$ & $\begin{array}{l}\text { Dos actores públicos } \\
\text { Agentes del sector público } \\
\text { involucrados }\end{array}$ \\
\hline $\begin{array}{l}\text { Fuente de información de } \\
\text { los habitantes locales }\end{array}$ & $\begin{array}{l}\text { Tres actores cívicos } \\
\text { Representantes de colecti- } \\
\text { vos defensores del centro }\end{array}$ \\
\hline $\begin{array}{l}\text { Fuente de información de } \\
\text { los desarrolladores }\end{array}$ & $\begin{array}{l}\text { Cinco actores privados } \\
\text { Arquitectos vinculados al } \\
\text { desarrollo y la construcción } \\
\text { de proyectos en el centro }\end{array}$ \\
\hline $\begin{array}{l}\text { Fuente de información de } \\
\text { un medio de comunicación }\end{array}$ & $\begin{array}{l}\text { Un actor externo } \\
\text { Periodista dedicado a docu- } \\
\text { mentar temáticas relativas a } \\
\text { desarrollo cultural del centro }\end{array}$ \\
\hline
\end{tabular}

Fuente: elaboración propia.

actores clave, al identificar elementos discursivos sobre percepciones del estado urbanístico actual (Masso, 2007). De esta forma, las narrativas se definen "como lo que los actores sociales de la vida urbana están diciendo sobre la ciudad o el barrio donde viven» (Mercier, 2008: 3). 
A partir de los elementos descritos en la tabla 4, se estructuran las entrevistas aplicadas al caso de estudio. El proceso metodológico implementado se describe en la tabla 5, y los resultados obtenidos configuran un diagnóstico sobre la situación actual del centro.

\section{Caso de estudio: Área Metropolitana de Monterrey (AMM) y su centro metropolitano}

El AMM es la tercera más grande de México y está conformada por 12 municipios, con más de 4 millones de habitantes en 2016. Es reconocida como la puerta comercial e industrial de México con el mundo por su ubicación geográfica, siendo un enclave logístico, industrial y de negocios. El crecimiento del AMM se caracteriza por la poca regulación y la falta de planeamiento urbano adecuado (García Ortega, 2001). Este factor, sumado a la falta de continuidad entre administraciones, ha provocado graves problemas urbanos.

Durante décadas, el centro de Monterrey ha sufrido pérdida de población, lo que se traduce en poca complejidad urbana. Los servicios y comercios predominan sobre la vivienda, hecho que contribuye a su deterioro material.

Figura 1. Cartografía de localización de Monterrey y su centro respecto al área metropolitana

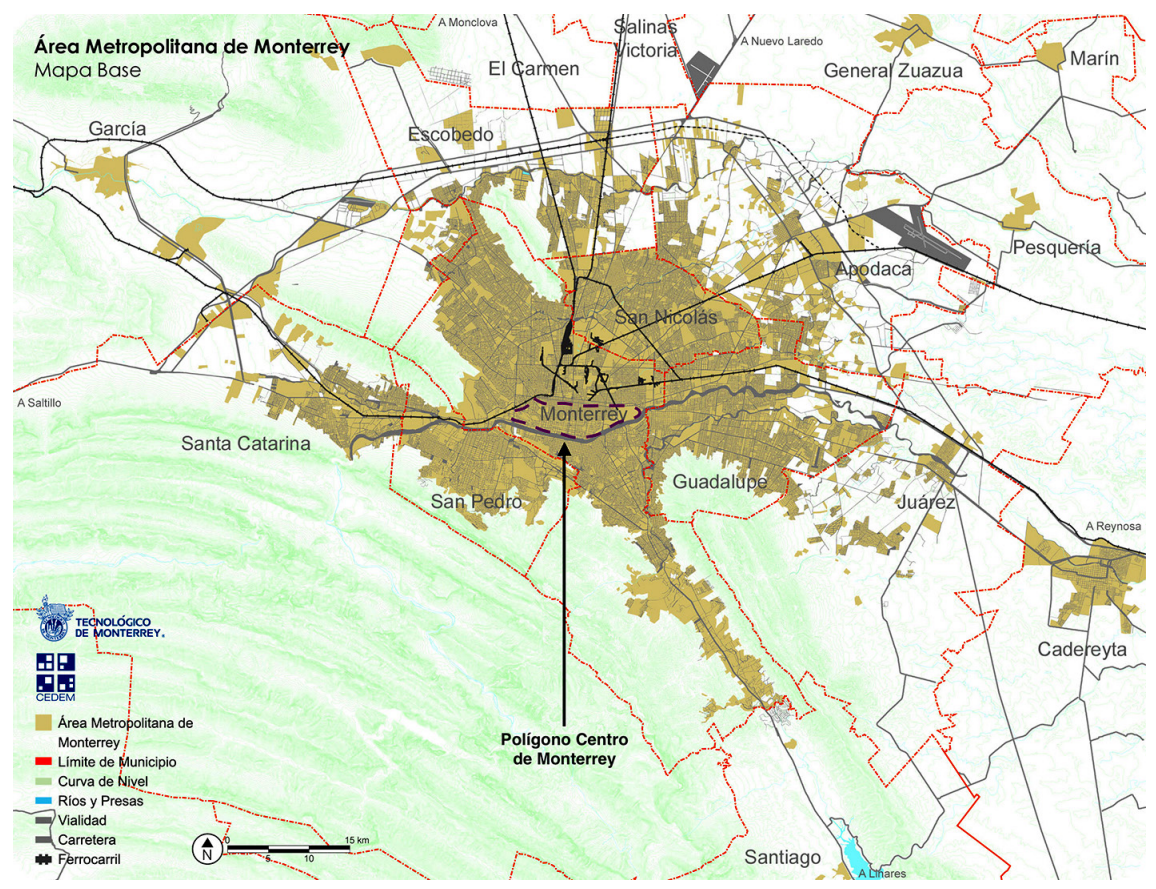

Fuente: CEDEM (2006). 
A partir de la década de los ochenta, el gobierno estatal comienza a intervenir urbanísticamente en el centro, con el objetivo de modernizarlo para promover la inversión privada y transformarlo en un distrito de negocios (Leal Iga y Fitch Osuna, 2012).

\subsection{Identificación sociourbanistica del centro de Monterrey}

Los límites del centro de Monterrey se componen de una superficie aproximada de 1.100 hectáreas, donde habitan alrededor de 30.000 habitantes (Garza-Rodríguez, 2015). La densidad de población del centro es de 27 hab./ha y cuenta con 1.502 viviendas en estado de abandono ${ }^{1}$. En los años sesenta, el centro empieza a perder población debido a la migración de las clases altas hacia la periferia (Prieto González, 2011). Este proceso se mantiene hasta finales de los setenta, cuando con el objetivo de revertir esta situación se ve la necesidad de transformarlo para atraer vivienda e inversión. Sin embargo, a pesar de los esfuerzos por regenerarlo, a día de hoy el centro sufre fenómenos de despoblación, abandono y degradación, así como una marcada segregación socioespacial (Aparicio et al., 2011), lo que contrasta con la imagen que el sector público y privado proyectan al mundo sobre el centro de la ciudad.

\subsection{Revitalización urbanistica del centro de Monterrey}

Se diferencian cuatro etapas en la regeneración del centro de Monterrey. Las tres primeras (1979-1986, 1991-1996 y 2003-2009) se caracterizan por la apuesta del sector público por la construcción de proyectos emblemáticos que promuevan la imagen de la ciudad. Las etapas coinciden con los periodos sexenales de la administración estatal. La última etapa, de 2013 a la actualidad, se caracteriza por formas emergentes de regeneración a partir de proyectos realizados por el sector público e intervenciones urbanas implementadas por residentes locales (figura 2 ).

Figura 2. Descripción general de las etapas de la intervención urbana

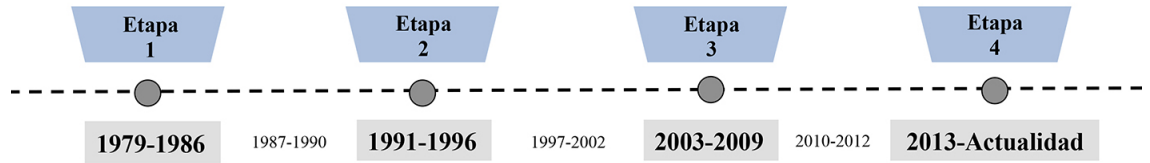

Fuente: elaboración propia.

1. Según un estudio realizado por el sector publico municipal. Acceso a través de notas de prensa: <http://www.elhorizonte. $\mathrm{mx} /$ local/en-el-abandono-edificios-delcentro-regio/799061>. 
Figura 3. Proyectos de regeneración urbana

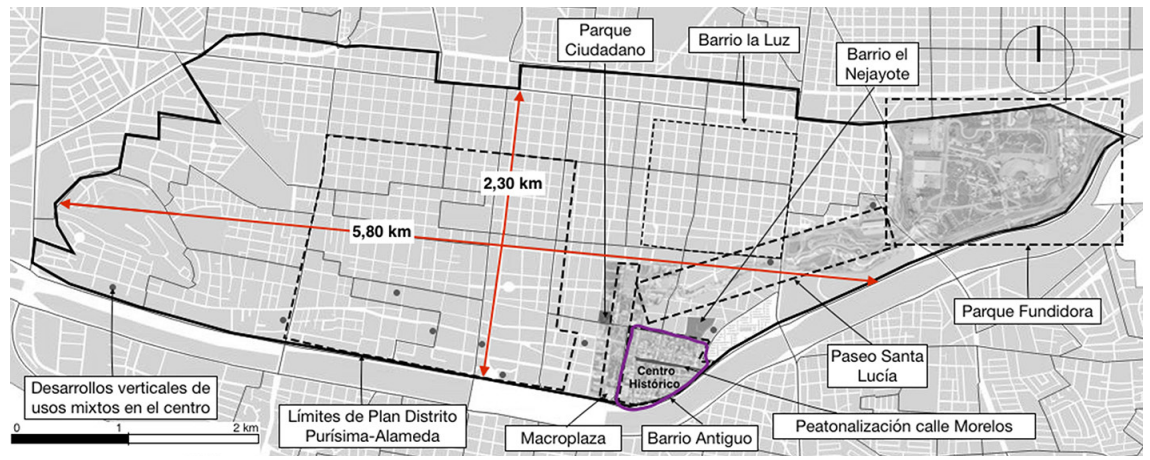

Fuente: elaboración propia.

\section{Etapa I - Proyectos emblemáticos}

Esta etapa empieza en la década de los ochenta, desde un modelo top-down, y está enfocada en promover proyectos emblemáticos «con más sentido estético que funcional» (Prieto González, 2011: 171) y en crear espacio público y equipamientos culturales. Parte de una iniciativa pública top-down (Leal Iga y Fitch Osuna, 2012), con la construcción de la Gran Plaza (Macroplaza), inaugurada en 1984, para impulsar la inversión privada y convertir la zona en centro financiero y de negocios (Prieto González, 2011) (figuras 4 y 5). Para realizar la Macroplaza, se derrumban 40 hectáreas construidas, lo que implica la reubicación de 283 familias y 310 negocios (Prieto González, 2011). El plan inicial de convertir la zona en un distrito de negocios no prospera debido a una crisis económica. De manera paralela, se proyecta un distrito financiero en otra zona del AMM que, al contar con un precio de tierra más barato, resulta más atractivo para los inversores (Prieto González, 2011). Esa zona es la que hoy se conoce como Valle Oriente, ubicada en el municipio de San Pedro Garza García.

\section{Etapa II - Barrio Antiguo y Ley de Patrimonio}

Al terminar la Macroplaza, se interviene en Barrio Antiguo, polígono que concentra inmuebles del siglo XVIII. El proyecto tuvo una duración de cinco años (1989-1994) y contó con financiamiento de los tres niveles de gobierno: federal, estatal y municipal. La intervención mejoró la estética del barrio tradicional con industrias culturales, de ocio y entretenimiento (Salgado Gómez, 2006; Prieto González, 2016), y con la concentración de equipamientos culturales de iniciativa privada (flagship projects), como el Museo de Arte Contemporáneo (MARCO) o el Museo de Historia Mexicana.

Como parte de la regeneración, se redacta la Ley de Patrimonio Cultural del Estado de Nuevo León, que entra en vigor a finales de 1991. Declara a Barrio Antiguo como zona protegida (1993) y lo denomina como centro 
Figuras 4 y 5 . Izquierda: maqueta sobre cómo se vería la Macroplaza (vista de norte a sur); derecha: macroplaza en 2009 (vista de norte a sur)
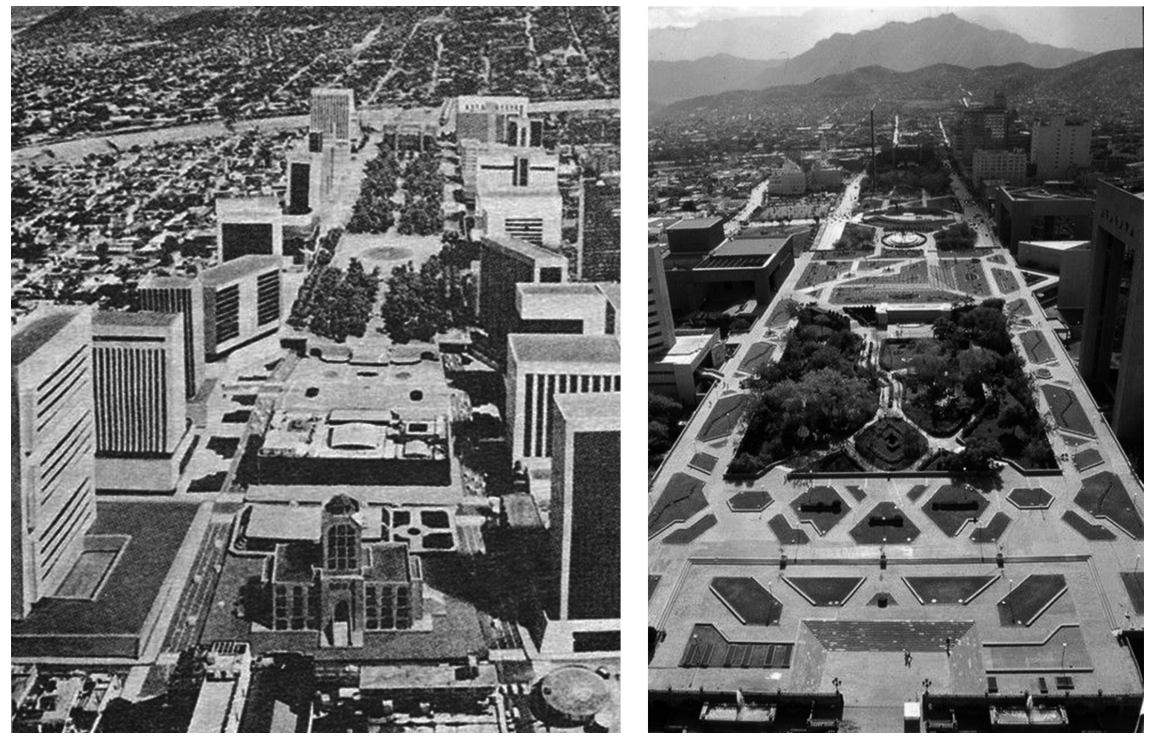

Fuente: izquierda: Fermín Téllez; derecha: Turismo Nuevo León.

histórico de la ciudad de Monterrey (Salgado Gómez, 2006; Prieto González, 2011). Hoy en día, la legislación que protege el patrimonio cultural del barrio sigue vigente, aunque se siguen produciendo intervenciones y demoliciones en inmuebles históricos catalogados sin que haya consecuencias legales.

\section{Etapa III - Espacio público, equipamientos y eventos}

En 1996, finaliza la construcción de la primera etapa del paseo Santa Lucía, intervención que incluye equipamiento cultural (Museo de Historia Mexicana) y espacio público (Plaza 400 Años y el paseo de Lanchitas) (Ovalle, 2007). La idea de conectar la Macroplaza y el Museo de Historia Mexicana con los terrenos de la Fundidora mediante un parque lineal estuvo interrumpida durante nueve años. En 2005, se realiza un plan de regeneración, el cual coincide con la preparación de la ciudad para el Fórum Universal de las Culturas, que se lleva a cabo en 2007. La segunda etapa del proyecto une 2,5 kilómetros (MacroplazaParque Fundidora) a través de un canal de agua artificial que los conecta, lo que impulsa la inversión privada en los alrededores (figura 4).

El proyecto del parque Fundidora surge de intervenciones top-down en el polígono y predios aledaños coordinadas con el sector privado, con el fin de incrementar la oferta turística, cultural, de ocio y recreación. Los vestigios de la antigua fábrica se ubican al noreste del centro, terreno de 231 hectáreas que al poco tiempo de su cierre, en 1988, es cedido al sector público. Las 
Figura 6. Paseo Santa Lucía y desarrollos inmobiliarios

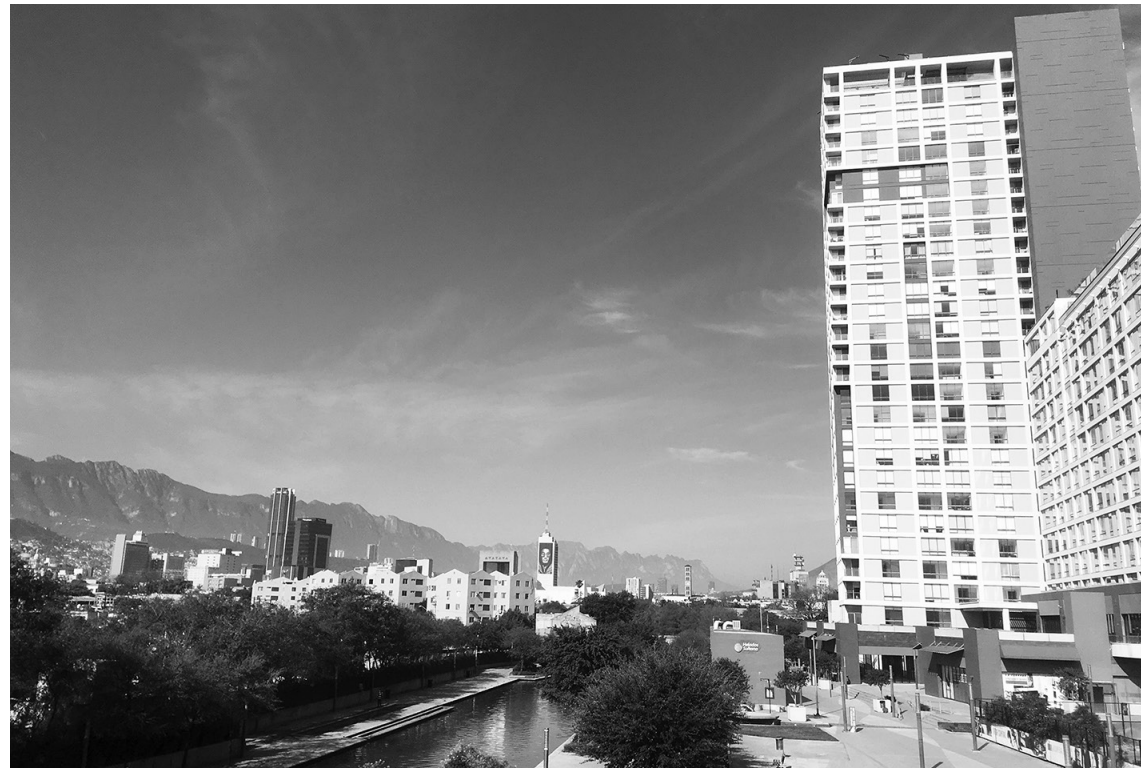

Fuente: fotografía de Fabiola Garza-Rodríguez (enero de 2018).

Figura 7. Naves industriales convertidas en equipamiento cultural dentro del parque Fundidora

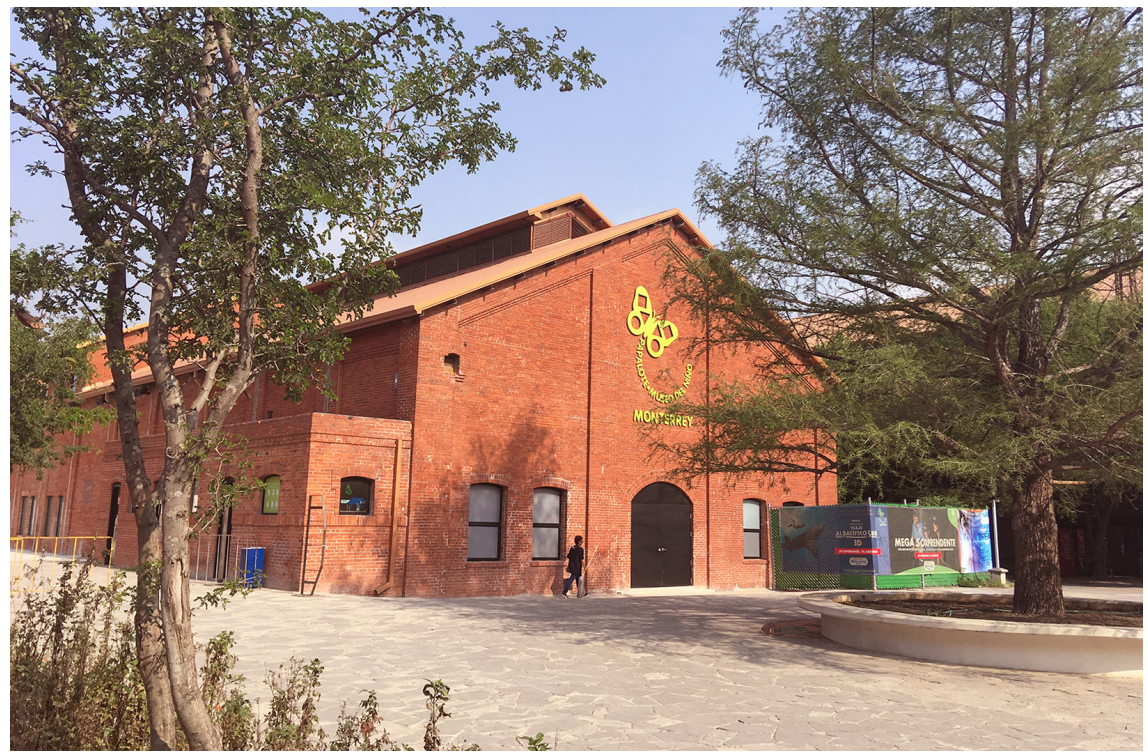

Fuente: fotografía de Fabiola Garza-Rodríguez (abril de 2018). 
Tabla 6. Síntesis de iniciativas de regeneración urbana en el centro de Monterrey

\begin{tabular}{|c|c|c|c|}
\hline Gobernanza & Gestión & Proyecto & Objetivo \\
\hline $\begin{array}{l}\text { Etapa I 1979-1986 } \\
\text { Top-down }\end{array}$ & Pública & $\begin{array}{l}\text { Construcción Gran } \\
\text { Plaza (Macroplaza), } \\
1984\end{array}$ & $\begin{array}{l}\text { - Impulsar la inversión } \\
\text { privada } \\
\text { - Convertir la zona en centro } \\
\text { financiero y de negocios }\end{array}$ \\
\hline
\end{tabular}

Descripción

Etapa II 1991-1996 Pública

Top-down

Intervención Barrio
Antiguo (1989-1994)

- Generar distritos culturales, concentración de actividades -industria cultural, creativa y artística

- Presencia de un ejemplar arquitectónico de marca

Fase I del paseo Santa Lucía
- Generar distritos culturales - Equipamientos culturales: a partir de la concentración Museo de Historia Mexicana de actividades relacionadas • Espacio público: con la industria cultural, creativa y artística Plaza 400 Años y lago con paseo de Lanchitas

\begin{tabular}{lll}
\hline Etapa III 2003-2009 & Público-privada & Fase II del paseo \\
Top-down & & Santa Lucía
\end{tabular}
Top-down

\section{- Creación de espacio público y mezcla de usos}

- Monterrey posmoderno: Construcción de proyectos emblemáticos «con más sentido estético que funcional» (Prieto González, 2011). Ejemplo: París, Nueva York

- Proyectos emblemáticos: Museo de Arte Contemporáneo (Marco)

- Planes regulatorios: Ley de Patrimonio Cultural del Estado de Nuevo León

Espacio público: Lago con paseo de Lanchitas

- Mezcla de usos: Renovación con vivienda, oficinas y comercio

\begin{tabular}{lll}
\hline Público-privada & Fase I del plan & - \\
& de la Fundidoración de espacio & público, equipamientos \\
& & culturales y recuperación \\
& de patrimonio industrial \\
& - Impulsar la inversión \\
& privada. \\
& - Convertir la zona en centro \\
& financiero y de negocios
\end{tabular}

- Espacio público

Parque Fundidora (2001) - Sitio de Arqueología Industrial Parque de diversiones infantil y de béisbol infantil

- Equipamientos culturales: Antiguas edificaciones restauradas Naves industriales reconvertidas en centros de arte Salas de conciertos

- Teatro al aire libre Proyectos arquitectónicos: Hotel de 5 estrellas Centro de negocios

\begin{tabular}{|c|c|c|c|}
\hline Pública & Macroevento & $\begin{array}{l}\text { Incluir agendas culturales } \\
\text { apoyando regeneración } \\
\text { urbana }\end{array}$ & $\begin{array}{l}\text { - Eventos } \\
\text { Fórum Universal de las Cultu- } \\
\text { ras } 2007\end{array}$ \\
\hline Público-privada & $\begin{array}{l}\text { Fase II del plan } \\
\text { de la Fundidora }\end{array}$ & $\begin{array}{l}\text { - Impulsar la inversión } \\
\text { privada } \\
\text { - Convertir la zona en centro } \\
\text { financiero y de negocios }\end{array}$ & $\begin{array}{l}\text { - Espacio público: } \\
\text { Pista de hielo, embarcadero y } \\
\text { áreas de esparcimiento } \\
\text { - Equipamientos culturales: } \\
\text { Museos, centro de exposi- } \\
\text { ciones } \\
\text { - Equipamientos de servicio: } \\
\text { Macroestacionamiento }\end{array}$ \\
\hline
\end{tabular}

\footnotetext{
Fuente: elaboración propia.
} 
intervenciones se materializan con la restauración de antiguas edificaciones y la reconversión de naves industriales en centros culturales.

$\mathrm{Al}$ proyecto se le suman un centro de negocios, sala de concierto, teatro al aire libre, un hotel de 5 estrellas, un parque de diversiones y un parque de béisbol infantil. En 2001, se da la apertura al parque, bajo la denominación de Sitio de Arqueología Industrial. En el periodo 2003-2009, se construye la segunda fase del proyecto, con la suma de 28 hectáreas en zonas recreativas (pista de hielo, embarcadero y áreas de esparcimiento), culturales (museos, centro de exposiciones) (figura 7) y de servicio (macroestacionamiento).

\subsection{Nuevas formas de regenerar el territorio: proyectos de escala local (2013-actualidad)}

\section{Etapa IV-Intervenciones alternativas}

A partir de 2013, surgen otros modelos de regeneración, desde el sector público (top-down) y desde el sector civil (bottom-up). Como ejemplo de uno público está la regeneración de la calle Morelos, en Barrio Antiguo, que impulsa la vocación cultural e histórica del barrio a partir de la peatonalización. Inaugu-

Figura 8. Peatonalización de la calle Morelos en Barrio Antiguo y Mercado Gourmet

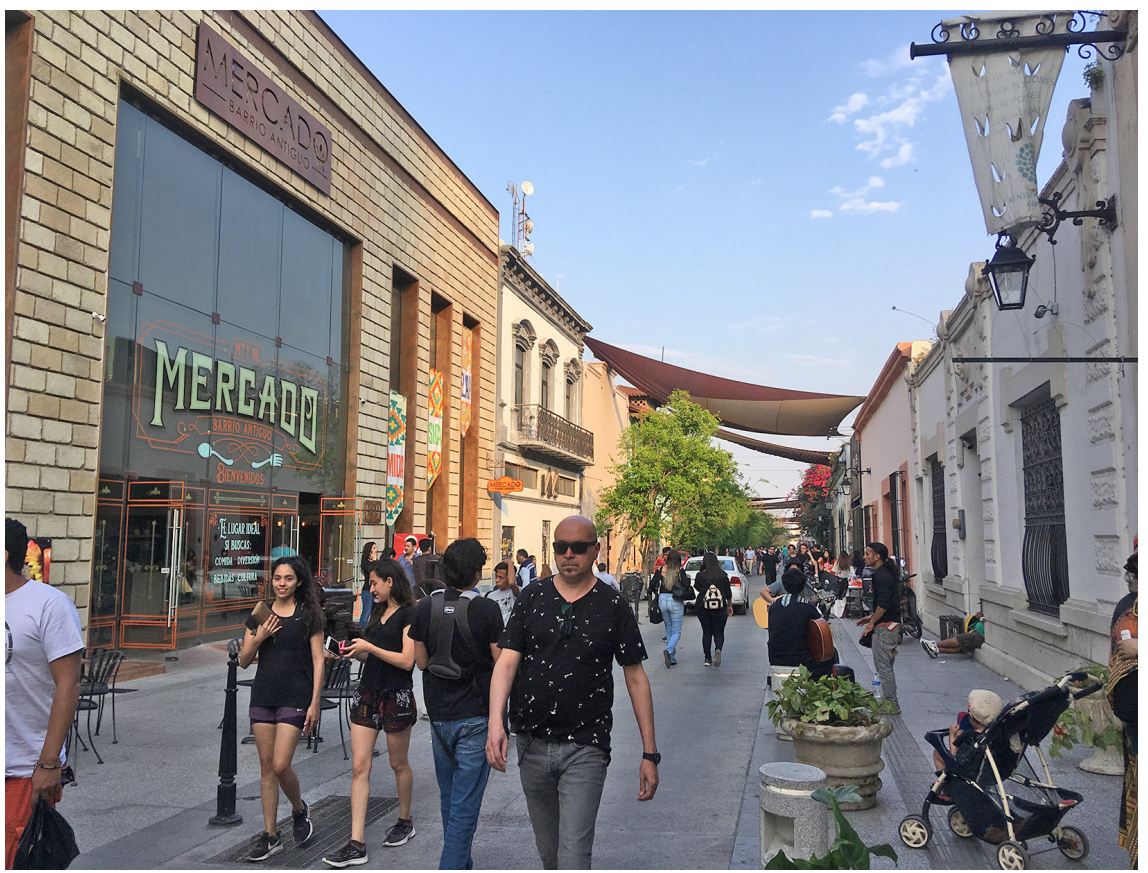

Fuente: fotografía de Fabiola Garza-Rodríguez (abril de 2018). 
rada a finales de 2014, las mejoras llevadas a cabo consisten en la nivelación de la calle, la ampliación de aceras, la instalación de bolardos y la arborización de especies nativas (figura 8). Se crearon bahías de ascenso y descenso, con acceso a internet gratuito. Sin embargo, los pocos residentes del polígono están en contra del proyecto, ya que perciben que propicia actividades de ocio nocturno que irrumpen en su vida cotidiana.

Otro proyecto público es el plan Distrito Purísima-Alameda, ubicado en el suroeste del centro. Su objetivo es fomentar inversiones mediante el desarrollo de áreas comerciales, habitacionales y de servicios. Se encuentra en fase de consulta (2019) para su gestión a través de alianzas entre diferentes actores (público, privado, académico y civil).

Otras estrategias urbanístico-culturales surgen desde un enfoque bottom-up, con impactos positivos en la sociedad. Una está en el Barrio del Nejayote, polígono ubicado al sureste de Barrio Antiguo. Durante años, fue foco de delito y criminalidad. Preocupados, residentes locales con vocación y profesión artística crean el colectivo y el proyecto Caminando en mi Barrio, para rehabilitar y mejorar las aceras con arte. Artistas y vecinos diseñan, financian e «intervienen» las aceras con mosaicos de colores, mediante la técnica conocida como trencadís, donde representan imágenes alusivas a la identidad colectiva de la ciudad (figura 9). El material proviene de donaciones y de medios económicos de los propietarios. El proyecto y colectivo ha crecido y lleva a cabo actividades

Figura 9. Intervención en Barrio del Nejayote del colectivo Caminando en mi Barrio

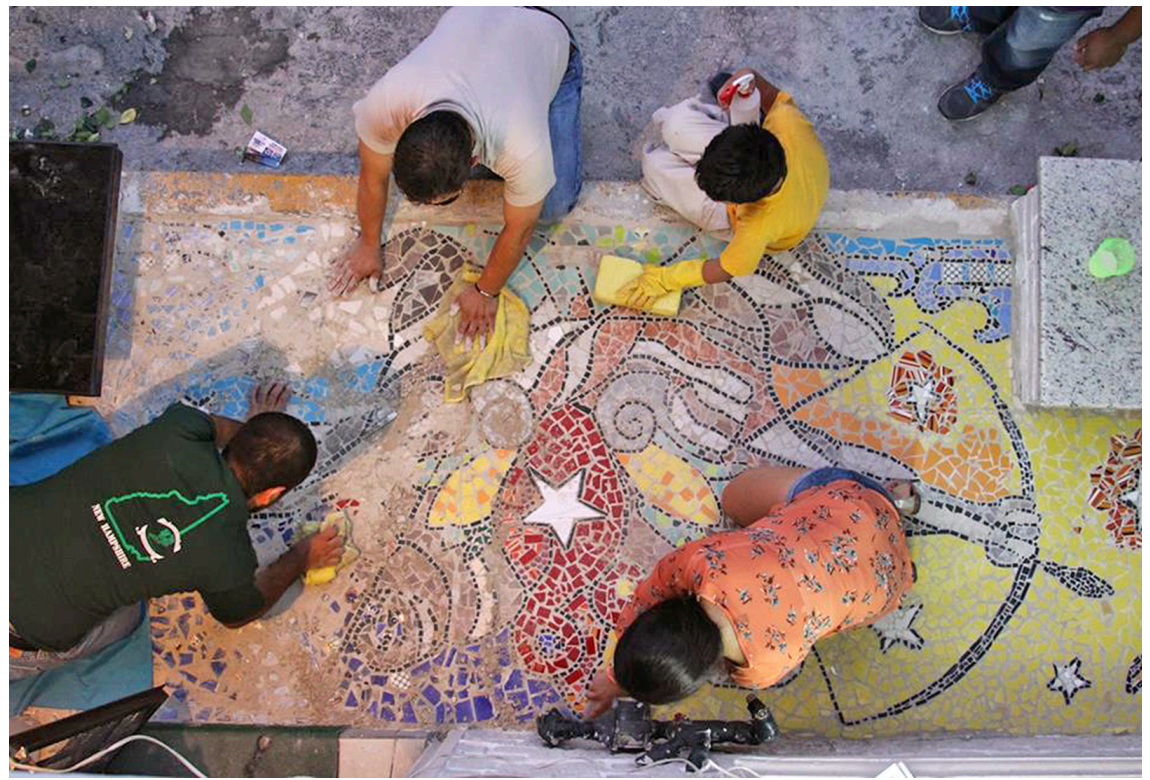

Fuente: Colectivo Caminando en mi Barrio. 
culturales como festivales de música, bazares y talleres artísticos. Además de mejorar la estética del lugar, fomenta el sentido de pertenencia y atrae turismo a la zona (Díaz-Fernández y Ledesma-Gómez, 2018).

Otro proyecto es el de Barrio La Luz, ubicado al noreste del centro, conocido como el barrio de artes y oficios. En 2013, se comienzan a asentar en esta zona estudios de artistas, talleres, escuelas de arte y cafés culturales, todos de nuevos vecinos. Así nace el colectivo Barrio La Luz Cultura Viva, como un grupo conformado por artistas y artesanos vecinos del barrio. El colectivo genera proyectos como festivales, actividades y manifestaciones culturales que promueven la identidad, el sentido de pertenencia y la cohesión social. La institución cultural del sector público - el Consejo para la Cultura y las Artes de Nuevo León (Conarte) - les otorga una beca económica por seis meses en 2017 para seguir realizando proyectos y actividades culturales. Esta intervención constituye un ejemplo de cómo iniciativas bottom-up trascienden a la esfera pública.

Otra iniciativa bottom-up es la del parque Ciudadano. A finales de 2014, el gobierno estatal anuncia la construcción de un estacionamiento destinado a diputados locales en un predio cercano a la Macroplaza. Ciudadanos y academia se manifiestan en contra del proyecto, con el argumento de la falta de áreas verdes, además del valor ecológico del solar — que contiene más de

Tabla 7. Síntesis de iniciativas de revitalización urbanística

\begin{tabular}{|c|c|c|c|c|}
\hline \multicolumn{5}{|c|}{ Etapa IV 2013 - Actual } \\
\hline Gobernanza & Gestión & Proyecto & Objetivo & Descripción \\
\hline \multirow[t]{2}{*}{ Top-down } & Pública & $\begin{array}{l}\text { Intervención Barrio } \\
\text { Antiguo } \\
\text { - Calle Morelos }\end{array}$ & $\begin{array}{l}\text { - Impulsar la } \\
\text { vocación cultural } \\
\text { e histórica del barrio }\end{array}$ & $\begin{array}{l}\text { - Espacio público: } \\
\text { Peatonalización de la calle More- } \\
\text { los (2014) }\end{array}$ \\
\hline & $\begin{array}{l}\text { Pública hacia } \\
\text { alianzas }\end{array}$ & $\begin{array}{l}\text { Plan Distrito } \\
\text { Purísima-Alameda }\end{array}$ & $\begin{array}{l}\text { - Revitalizar el distrito } \\
\text { con mezcla de usos } \\
\text { - Impulsar la inversión } \\
\text { privada }\end{array}$ & $\begin{array}{l}\text { - Mezcla de usos: } \\
\text { Renovación con vivienda, oficinas } \\
\text { y comercio } \\
\text { - Gestión colaborativa: } \\
\text { Modelo de planeamiento colabora- } \\
\text { tivo entre actores del sector públi- } \\
\text { co, privado, académico y civil }\end{array}$ \\
\hline \multirow[t]{3}{*}{ Bottom-up } & $\begin{array}{l}\text { Iniciativa residentes } \\
\text { colectivo }\end{array}$ & $\begin{array}{l}\text { Caminando en mi Barrio, } \\
\text { Barrio del Nejayote }\end{array}$ & $\begin{array}{l}\text { Rehabilitar y mejorar } \\
\text { de manera estética las } \\
\text { aceras del barrio }\end{array}$ & $\begin{array}{l}\text { - Espacio público: } \\
\text { Intervenciones artísticas en aceras }\end{array}$ \\
\hline & $\begin{array}{l}\text { Iniciativa residentes } \\
\text { colectivo Barrio La } \\
\text { Luz Cultura Viva } \\
\text { *Apoyo posterior } \\
\text { sector público (2017) }\end{array}$ & Barrio La Luz & $\begin{array}{l}\text { Promover la identidad } \\
\text { del barrio, el sentido de } \\
\text { pertenencia y la cohesión } \\
\text { social entre vecinos }\end{array}$ & $\begin{array}{l}\text { Eventos: } \\
\text { Festivales, actividades y manifes- } \\
\text { taciones culturales }\end{array}$ \\
\hline & $\begin{array}{l}\text { Iniciativa ciudadana } \\
\text { okupan }\end{array}$ & Parque Ciudadano & $\begin{array}{l}\text { - Crear un espacio } \\
\text { público para la } \\
\text { ciudadanía }\end{array}$ & $\begin{array}{l}\text { - Espacio público: } \\
\text { Manifestación y preservación del } \\
\text { verde urbano y creación de un } \\
\text { parque }\end{array}$ \\
\hline
\end{tabular}

Fuente: elaboración propia. 
Figura 10. El parque Ciudadano recién inaugurado

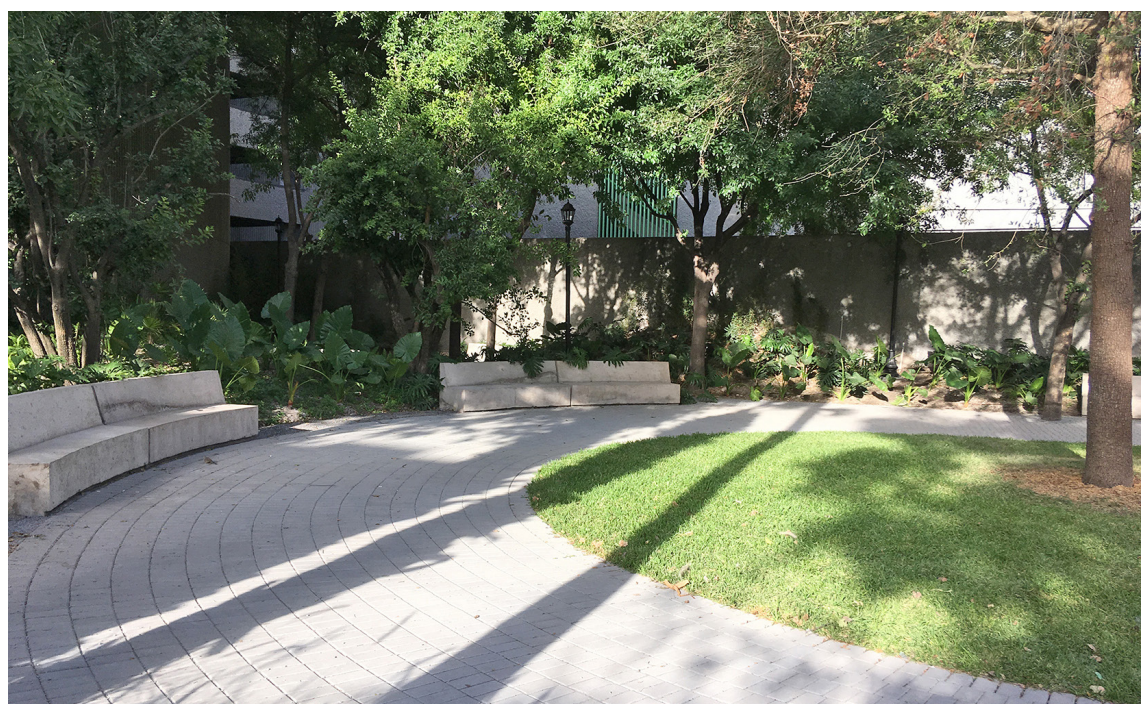

Fuente: fotografía de Fabiola Garza-Rodríguez (abril de 2018).

30 árboles de la región—, lo que es motivo suficiente para detener la construcción. A modo de presión, ciudadanos okupan el solar durante 14 días ininterrumpidos y logran su objetivo.

Las negociaciones ciudadanas para que el solar se convierta en parque duran más de dos años, durante los cuales la ciudadanía lo utiliza como espacio público. Además, la gente se organiza para realizar acciones de limpieza, mantenimiento y ocupación. Una vez aprobada la construcción del parque, el diseño se convoca a concurso público. A finales de 2017 , se inicia la intervención del proyecto ganador, con solo el $1 \%$ del presupuesto asignado para el estacionamiento. En abril de 2018, se inaugura (figura 10) y se convierte en un hito histórico por su concreción gracias a la participación ciudadana.

\section{Narrativas a partir de las intervenciones urbanísticas en torno al centro}

A continuación, se presentan las narrativas obtenidas en torno al centro a modo de resultados, para ayudar a clarificar el diagnóstico y la percepción social sobre el estado urbanístico del centro de Monterrey.

\subsection{El juicio: Las problemáticas urbanas y su origen}

Una narrativa común entre los entrevistados manifiesta que el problema principal es el estado de abandono de los inmuebles del centro. Este abandono responde al vacío legal cuando el inmueble se queda prácticamente sin dueño, ya que los propietarios mueren sin hacer testamento. Cuando 
Figura 11. Inmuebles en condiciones de abandono y degradación en el centro

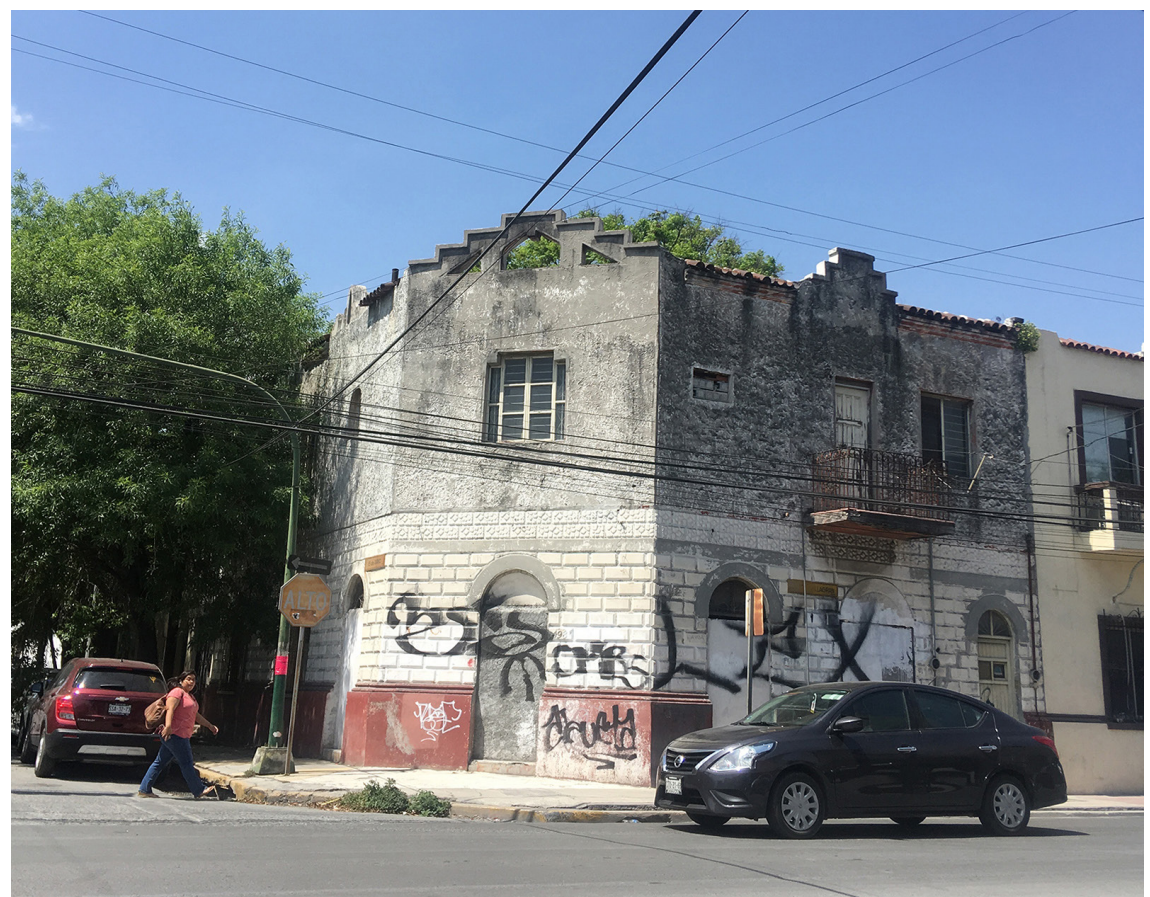

Fuente: fotografía de Fabiola Garza-Rodríguez (abril 2018).

un inmueble queda intestado, entra en un ciclo de procesos legales largos y costosos (figura 11).

Es común encontrar edificaciones abandonadas y con deterioro material, lo que expone al centro como un lugar no habitable. Representantes de colectivos dicen que el problema es de percepción, que el abandono no es una realidad. Activistas e investigadores concuerdan que este fenómeno no es generalizado ni extrapolable al resto del centro. Defienden que esta percepción se debe a la falta de mantenimiento urbano en ciertas zonas por parte del sector público.

Hacen falta equipamientos públicos, culturales, espacios de recreación, áreas verdes y servicios básicos, ya que la ausencia de complejidad urbana se muestra como una limitación para la constitución de un centro compacto, accesible y cohesionado. El sector público y privado muestran preferencia por desarrollar otros sectores del AMM y lo justifican diciendo que no hay incentivos económicos y fiscales para el desarrollo inmobiliario del centro.

Adicionalmente, la complejidad de los trámites oficiales para construir o remodelar un inmueble en el centro se vuelve un proceso largo y complicado, lo que desmotiva al sector privado. La narrativa de un actor del sector público es que la autoridad pone el foco en «inventar» normas y estudios que no necesita la propiedad privada, lo que se convierte en un limitante para su desarrollo. 
Figura 12. Casona norestense del siglo XVIII, catalogada como patrimonio arquitectónico, en el centro a medio derruir

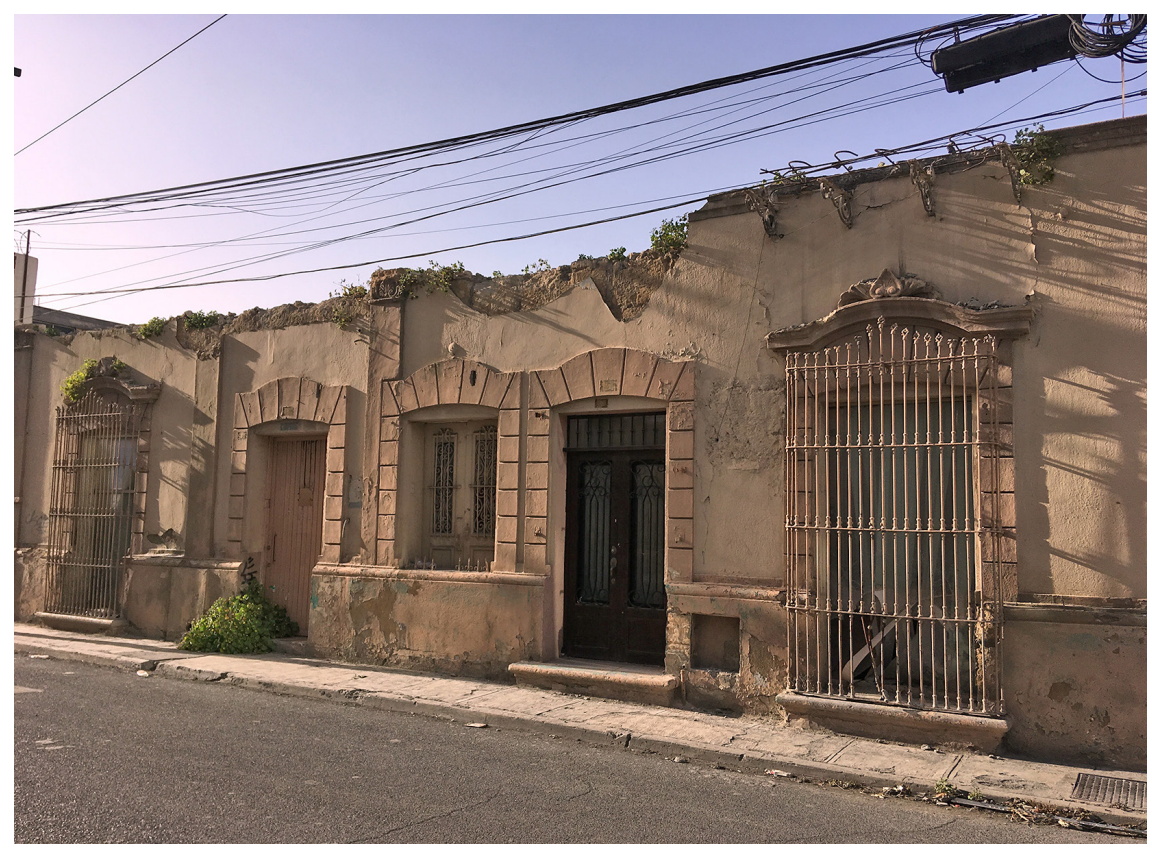

Fuente: fotografía de Fabiola Garza-Rodríguez (diciembre de 2017).

La postura es que las problemáticas recaen en el gobierno, por ser responsable de crear políticas de construcción y mantenimiento que favorecen la especulación urbanística (Harvey, 2005) y gentrificación (Zukin, 1987). Sin embargo, el sector privado también tiene responsabilidad al intervenir inmuebles con fines especulativos, ya que se manifiesta en la compra/venta de inmuebles en mal estado para intervenirlos y buscar un mayor beneficio económico con su venta. Este fenómeno es visible en casi todo el centro de Monterrey, donde inmuebles abandonados son convertidos en comercios de lujo, fenómeno identificado por Carrión (2007) como "boutiquización», donde el uso de suelo residencial es modificado a uso comercial (RiusUlldemolins y Posso Jiménez, 2016), cosa que altera, además, patrones de consumo.

En el centro existen edificaciones de alto valor patrimonial que datan de los siglos XVIII, XIX y principios del XX, además de edificios representativos del movimiento moderno y art déco. Sin embargo, el desconocimiento atenta contra su preservación, y puede eliminar de manera arbitraria el pasado (figura 12), causando procesos de gentrificación simbólica (Janoschka y Sequera, 2014; Delgadillo, 2009), a través de intervenciones exclusivas para turistas y ciertas élites, lo que limita tanto la tipología arquitectónica como el tipo de usuario. 
Este tipo de gentrificación es visible en el Barrio Antiguo (Moreno Zúńiga y Jurado Montelongo, 2018).

\subsection{El proyecto: las actuaciones urbanisticas}

Investigadores y activistas declaran que son pocas las actuaciones profundas que se han hecho para regenerar el centro. Según su opinión, los proyectos del sector público se realizan de manera impositiva y sin participación ciudadana.

No es un problema de que el proyecto este mal planteado, que a veces sí, pero no es el principal. El problema es que no hay negociaciones entre partes, no hay diálogos, no hay mesas de debate, no hay esto. (Arquitecto)

Por otro lado, el sector público ha realizado proyectos de mejora urbana, como la peatonalización de la calle Morelos. Más allá de estas intervenciones, están en proceso planes de regeneración enfocados en polígonos específicos (por ejemplo, el plan Distrito Purísima-Alameda, publicado a finales de 2017), cuyo fin es continuar con la ardua tarea de revitalizar el centro.

Estamos enfocados en darle mayor calidad de vida al peatón en el centro de Monterrey, esto es mejorar las banquetas, mejorar la escala humana de la ciudad. [...] Para el centro, la visión es recuperar el espacio público. Sentimos que si el espacio público es de calidad, vendrá la inversión, y entonces vendrán todas esas obras nuevas, esa es nuestra apuesta, crear más espacio público, y en paralelo una visión metropolitana. (Representante de la Administración pública municipal)

La narrativa del sector público muestra interés por dotar a los habitantes de espacios de calidad. Pero las entrevistas identifican desconfianza hacia la autoridad por las malas prácticas, ya que en muchas ocasiones los proyectos nunca llegan a concretarse.

El sector público reconoce la falta de confianza de la comunidad en las instituciones, por lo que propone el modelo de planeación colaborativa entre actores (público, privado, académico y civil), con una aportación del Instituto Municipal de Planeación Urbana y Convivencia Monterrey (IMPLANcMTY). El planeamiento colaborativo gestiona proyectos con actores locales, ya que de esta forma residentes locales e inversores trabajan junto con el sector público, aunque sin saberlo formalmente. El primer caso de éxito de este modelo es Distrito Tec, en la zona sur de Monterrey. La apatía social hacia las instituciones sucede en todas partes del mundo, «la planificación se está enfrentando a una crisis de legitimidad mundial» (Miraftab, 2018: 220), por lo que es necesario aplicar nuevas alternativas para planear e intervenir el territorio.

Lo que se hizo fue decir «no me alcanza; pero traigo estas ideas, ¿cómo ven si...?». Y para que esto funcione necesito que este proyecto que estoy creando sea tuyo, porque sospecho que cuando me vaya me lo van a cortar, y ese fue el modelo. (Agente del sector público local [al hablar sobre Distrito Tec]). 
La percepción sobre proyectos bottom-up es positiva. Se ven como casos de éxito, porque algunos han sido apoyados por la esfera pública (como el parque Ciudadano). En ese sentido, es interesante la apertura del sector público - aunque mínima - hacia iniciativas sociales. Este modelo es el que Healey (2003) define como planeación colaborativa, por generar intervenciones urbanísticas a través de acciones colaborativas. Las colaboraciones son relevantes en este contexto, ya que la participación ciudadana en iniciativas tradicionales es mínima, y el valor más potente de las actuaciones bottom-up es lograr transformaciones urbanas que promuevan gobernanzas colaborativas donde el ciudadano juega un rol clave (Healey, 2003; Geissel, 2008; Miraftab, 2018).

El proyecto del Nejayote es una iniciativa muy especial, que embellece el lugar y ha actuado como una bola de nieve beneficiando a los vecinos, porque todos quieren unirse y transformar sus aceras. [...] Ahora las personas reconocen el barrio y lo asocian inmediatamente con la intervención en las aceras. (Arquitecto)

Los proyectos top-down producen espacios y proyectos emblemáticos con impactos estéticos y de paisaje urbano. Teniendo en cuenta que la tipología urbanística ha sido una apuesta por mejorar la imagen del centro, hay oportunidades de mejora para superar los desafíos de los cambios de mandatarios locales involucrando de manera más directa a la comunidad con modelos más colaborativos o participativos (Blakeley, 2005; Rius-Ulldemolins y Posso Jiménez, 2016).

Los proyectos bottom-up transforman la imagen, la estética y la percepción a partir de intervenciones puntuales. Refuerzan la identidad local y el sentido de pertenencia a través de actividades y manifestaciones culturales. Sin embargo, la complejidad y la escala del AMM requiere de incentivos para que surjan más iniciativas y para que las existentes puedan consolidarse como procesos permanentes, con el involucramiento de otros actores (públicos, privados, académicos). En ese sentido, la investigación de Parés, Bonet-Martí y Martí-Costa (2012) demuestra los impactos positivos que la participación ciudadana genera como constructor de valores culturales entre residentes.

\subsection{El programa: potencialidades y propuestas en un centro olvidado}

El programa involucra lo que Mercier (2008) identifica como alternativas para alcanzar un objetivo. Una narrativa en común es el potencial de uso de recursos culturales en el centro como capital cultural (Bordieu, 1997): espacio público, áreas verdes, patrimonio histórico-arquitectónico, elementos simbólicos y equipamiento cultural son escasos, pero es necesario fomentar su uso y relación con el entorno urbano e integrarlos como activos en la vida cotidiana de los habitantes.

Aunque escasos, los equipamientos culturales son de calidad, como el Museo de Arte Contemporáneo (MARCO), el Museo de Historia Mexicana o el Teatro de la Ciudad. Sin embargo, estos se concentran en los alrededores 
de la Macroplaza. Su centralidad geográfica limita la proximidad y accesibilidad para el resto de la población del AMM. Otra limitante es la centralidad institucional que crea, distribuye y difunde la cultura, ya que, según narrativas, tiende a la exclusividad de los interesados.

Es medio underground, sí hay cosas, pero necesitas estar en el medio (cultural). Es la misma gente que se mueve de un lugar a otro, y son actividades más o menos parecidas unas a otras. No hay difusión para gente que no conozca o viva en el centro, y a veces hay cuestiones culturales interesantes y la difusión es mínima. (Activista)

Algunas narrativas sugieren que el rol de la cultura se manifiesta en el arte urbano, en la creación de espacios públicos y la diversificación de oferta cultural. Actores del sector público, privado y civil creen que la respuesta está en estrategias dirigidas a la representación de valores culturales, como la estética. El sector académico argumenta la necesidad de explorar el valor de inspiración de la cultura mediante expresiones artísticas, restauración de inmuebles patrimoniales o prácticas simbólicas y espirituales. Otras propuestas consideran la ocupación temporal de vacíos urbanos a través de actividades que promuevan la convivencia. La cultura puede ser estrategia de regeneración del centro porque, al fortalecer el sentido de pertenencia, es posible generar transformaciones de raíz que trasciendan hacia la esfera público-privada mediante regeneraciones colaborativas y de largo plazo.

El rol de la cultura es ayudarnos a reconocernos a nosotros mismos como ciudadanos de un lugar, reconocer que tenemos tradiciones, costumbres, que tenemos sueños más allá de la producción y el consumo. (Investigadora)

\section{Discusión: hacia regeneraciones urbanas colaborativas}

La cultura se posiciona como estrategia de regeneración urbana en la era de la globalización (Landry, 2000; García, 2004; Miles, 2005), ya que, además de atraer inversiones, permite la revalorización de la comunidad fomentando la cohesión social y proyectando una nueva imagen de ciudad sostenida en la creatividad, las artes, la innovación y las políticas culturales (Montgomery, 2003; Landry, 2003; Evans y Shaw, 2004; Florida, 2005; Bianchini y Ghilardi, 2007).

En Monterrey, se reconoce un proceso en el que inicialmente las regeneraciones fueron públicas, bajo modelos de gobernanza top-down, y donde posteriormente se dan desde modelos bottom-up.

Temporalmente identificamos cuatro etapas de experiencias urbanísticas en el centro de Monterrey:

1. Proyectos emblemáticos: En la primera etapa, de 1979 a 1986, inicia la regeneración con la creación de proyectos emblemáticos, estrategia cultural basada en la construcción y la reconversión de equipamientos e infraestructura cultural de gran escala urbana (Macroplaza), para posicionar la ciudad en el contexto global (Prieto González, 2011). 
2. Barrio Antiguo y Ley de Patrimonio: La segunda etapa, de 1991 a 1996, se enfoca en políticas que protegen el polígono considerado como centro histórico de la ciudad: el Barrio Antiguo. Los proyectos desarrollados desde modelos top-down son señalados por los entrevistados del sector civil y académico, ya que no han conseguido resultados en transformaciones sociales y culturales con su regeneración. Los beneficios económicos se dirigen a propietarios y al mercado inmobiliario dedicado al ocio nocturno. En otros contextos, estas prácticas producen más marginación y degradación, en lugar de atenuarla (Eizenberg y Cohen, 2015).

3. Espacio público, equipamientos y eventos: La tercera etapa, de 2003 a 2009, produce intervenciones urbanísticas asociadas a megaeventos y a la creación de equipamiento e infraestructura cultural. Esto se hace a partir de la creación y/o recuperación de antiguos inmuebles en espacios culturales, de ocio y entretenimiento, donde la cultura se liga al modelo de desarrollo urbano de la ciudad (Degen y García, 2012; Jeannotte, 2016). Sin embargo, se vuelve necesario descentralizar la cultura, ya que solo un organismo público la maneja de manera oficial en el Estado y limita su heterogeneidad y alcance (Zaid, 2013).

4. Intervenciones alternativas: La cuarta etapa corresponde al periodo de 2013 a la actualidad y se caracteriza por el surgimiento de iniciativas bottom-up, que han activado procesos de menor escala con la colaboración entre actores - como sociedad civil y sector público-, como en el caso del parque Ciudadano. En otros contextos, estas condiciones generan cohesión social porque tienden a la construcción de trabajo colaborativo entre instituciones y sociedad civil, de forma que promueven la sostenibilidad cultural del lugar (Truñó, 2008; Rius-Ulldemolins y Posso Jiménez, 2016; Lazarević et al., 2016).

La clasificación de las estrategias urbanísticas implementadas en los últimos 30 años muestra la evolución seguida por los sectores público, privado y civil. Por una parte, la regeneración, liderada por el sector público asociado con el privado, comienza a partir de intervenciones radicales como la tabula rasa en el tejido urbano. Las estrategias tienden a ir complementando la primera a partir de acciones orientadas a ofrecer o acercar la cultura a la ciudadanía. Esto es visible con la creación de museos, la recuperación de inmuebles industriales para convertirlos en parque cultural, y también con la creación o la recuperación de espacios públicos. De manera paralela pero más actual, las estrategias alternas a las oficiales para regenerar el centro surgen a partir de iniciativas culturales en el espacio urbano propuestas y gestionadas por los ciudadanos, y en ocasiones secundadas por el sector público-privado, lo que demuestra que las regeneraciones colaborativas son eficaces (Healey, 2003).

Los impactos generados a partir de las intervenciones realizadas son tanto positivos como negativos. Los impactos positivos ofrecen mejor calidad de vida a los habitantes, accesibilidad a espacios públicos y a la cultura, y ayudan en la transición hacia ciudades y comunidades sostenibles (Hawkes, 2001; Duxbury 
Tabla 8. Síntesis de elementos comparativos entre procesos de regeneración

\begin{tabular}{ll}
\hline Periodo & Gobernanza \\
\hline Etapa I: & Top-down \\
Proyectos & Liderada desde el sector \\
emblemáticos & público e implementada \\
$1979-1986$ & $\begin{array}{l}\text { a través de asociaciones } \\
\text { con el sector privado }\end{array}$
\end{tabular}

Estrategias urbanísticas Impactos

- Creación de espacio - Desplazamiento de público para convertirlo población

en nodo de negocios - Creación de espacios

- Reconversión de una públicos para la ciudaantigua fábrica danía y de museos de arquitectura industrial (patrimonio)

Etapa II: Top-down

Barrio Antiguo y

Ley de Patrimonio

1991-1996
- Regeneración urbana de Barrio Antiguo

- Creación de proyectos emblemáticos culturales para el centro

- Creación de primera parte del proyecto del paseo Santa Lucía

- Mejora escenográfica y cambio de uso de suelo para bares y restaurantes

- Creación de equipamientos culturales como museos, teatros y biblioteca

- Creación de espacio público, conexiones urbanas y peatonales

\begin{tabular}{|c|c|c|c|}
\hline $\begin{array}{l}\text { Etapa III: } \\
\text { Espacio público, } \\
\text { equipamientos y } \\
\text { eventos } \\
\text { 2003-2009 }\end{array}$ & $\begin{array}{l}\text { Top-down } \\
\text { Liderada desde el sector } \\
\text { público e implementada } \\
\text { a través de asociaciones } \\
\text { con el sector privado }\end{array}$ & $\begin{array}{l}\text { - Creación de la } \\
\text { segunda parte del } \\
\text { río que conecta dos } \\
\text { espacios públicos } \\
\text { - Sede de megaevento } \\
\text { internacional } \\
\text { - Creación de más } \\
\text { espacio público en } \\
\text { el parque Fundidora }\end{array}$ & $\begin{array}{l}\text { - Aumento de espacio } \\
\text { público para completar } \\
\text { el proyecto de conexión } \\
\text { Santa Lucía-parque } \\
\text { Fundidora } \\
\text { - Atención internacional } \\
\text { por ser sede de megae- } \\
\text { vento, aumento en el } \\
\text { turismo }\end{array}$ \\
\hline $\begin{array}{l}\text { Etapa IV: } \\
\text { Intervenciones } \\
\text { alternativas } \\
\text { 2013-actualidad }\end{array}$ & $\begin{array}{l}\text { Bottom-up } \\
\text { Liderada desde el sector } \\
\text { cívico (colectivos) e imple- } \\
\text { mentada con el apoyo } \\
\text { económico posterior del } \\
\text { sector público }\end{array}$ & $\begin{array}{l}\text { - Intervención en } \\
\text { aceras con técnica } \\
\text { artística } \\
\text { - Concentración de } \\
\text { artistas en un barrio } \\
\text { del centro } \\
\text { - Manifestación social } \\
\text { en contra de un } \\
\text { estacionamiento }\end{array}$ & $\begin{array}{l}\text { - Mejora en la estética } \\
\text { y percepción urbana } \\
\text { del barrio } \\
\text { - Aumento en cohesión } \\
\text { social, sentido de } \\
\text { pertenencia } \\
\text { - Creación de espacio } \\
\text { público }\end{array}$ \\
\hline
\end{tabular}

Fuente: elaboración propia.

et al., 2015). También se fortalecen valores culturales que identifican a la comunidad, como sentido de pertenencia, identidad y cohesión social (Evans y Foord, 2008; Stern y Seifert, 2010). Sin embargo, el precio es alto, ya que los proyectos donde interviene el sector público-privado tienden a favorecer al turismo o a ciertas élites (Delgadillo, 2009; Janoschka y Sequera, 2014), con los evidentes procesos de gentrificación, en lugar de promover la permanencia y el empoderamiento de residentes locales. Hacen falta proyectos públicoprivados que realmente revitalicen el centro, ya que los elementos culturales existentes no llegan a producir transformaciones urbanas significativas. Prácticas artísticas y culturales deberían ser reconocidas como parte integral del desarrollo sostenible en forma de políticas culturales. Sería interesante replicar 
en distintas áreas del centro de Monterrey estrategias culturales adaptadas a necesidades de cada barrio.

\section{Conclusión}

El trabajo analiza los proyectos de regeneración urbana implementados en el centro de Monterrey. Se reconoce que el sector público ha ejercido el liderazgo con la creación de espacios públicos y culturales que contribuyen a la imagen urbana. La falta de continuidad de proyectos públicos hace que las intervenciones se basen en infraestructura física, y esto deja de lado cuestiones sociales sin resolver. Es decir, la regeneración como un proceso de interacción entre actores para el fortalecimiento de las dinámicas culturales de largo alcance no ha contado con el mismo seguimiento. Por lo tanto, convertir el centro en un lugar dinámico, complejo y cohesionado para la ciudadanía sigue siendo una tarea en proceso.

La apuesta gubernamental se ha orientado hacia acciones culturales relativas a proyectos. La inversión pública enfocada en construir proyectos emblemáticos es un paso positivo de transformación urbana, sin embargo, la falta de comunicación entre actores y dependencias se muestra como uno de los principales limitantes para producir regeneraciones de fondo. Por ello, es fundamental trabajar de manera colaborativa entre actores, para producir regeneraciones más integrales. En este caso, el modelo bottom-up produce impactos positivos reflejados en la creación de actividades culturales y artísticas, y contribuye a la sostenibilidad cultural al fomentar actividades que refuerzan la identidad, la cohesión social y el sentido de pertenencia entre habitantes.

Para cumplir los propósitos de la regeneración en centros históricos, el despliegue de las estrategias culturales podría ser un elemento que genere cohesión social e identidad. Si bien no todas las estrategias son replicables, siempre es posible proponer intervenciones basadas en componentes locales para potenciar las fortalezas de sus actores. Se debe integrar en la práctica urbanística lo cultural, económico, social y ambiental (Healey, 2003). Se ha demostrado que contribuciones más holísticas y colaborativas (Healey, 1996; 2003; Miraftab, 2018) promueven mayores impactos y fomentan la sostenibilidad en la ciudad en todos los aspectos. Precisamente, este es el reto de las ciudades contemporáneas. Dentro de los aportes más significativos, se comprueba que los proyectos logran mayor consolidación al crear sinergias entre actores.

Es valioso contrastar narrativas para visibilizar oportunidades de mejora y lecciones aprendidas de décadas de intervenciones, donde además se manifiesta la falta de confianza que la sociedad civil tiene hacia las instituciones como uno de los principales limitantes para producir transformaciones de raíz. Aunque el sector público tenga buenas intenciones, al ser sus trámites largos y costosos, esto termina por jugar en contra del desarrollo. Las valoraciones apuntan a que los proyectos bottom-up producen impactos positivos, ya que transforman la imagen urbana y la percepción social, pero hace falta la vinculación entre actores para que sean relevantes. 
En un recuento sobre lo formulado, como propuesta hacia futuras regeneraciones urbanas, se sugiere apostar por un modelo de colaboración entre actores, donde sociedad civil, sector público y sector privado generen coaliciones y trabajen de manera paralela. En este caso, son una referencia los planteamientos de Miraftab (2018) sobre planeación colaborativa. Esto supondría una revisión y una mejora en las formas de intervenir en el territorio, a partir de procesos de planeación y gestión más flexibles, que incorporen otros ámbitos como punto base para producir regeneraciones urbanas más integrales y creativas.

\section{Referencias bibliográficas}

Aparicio Moreno, C. E.; Ortega Rubí, M. E. y Sandoval Hernández, E. (2011). «La segregación socio-espacial en Monterrey a lo largo de su proceso de metropolización». Región y Sociedad, 23 (52), 173-207. $<$ https://doi.org/10.22198/rys.2011.52.a185>

BAYLISS, Darrin (2004). «Creative planning in Ireland: the role of culture-led development in Irish planning». European Planning Studies, 12, 497-515. <https://doi.org/10.1080/0965431042000212759>

BECKER, A. y MÜLLER, M. M. (2013). «The securitization of urban space and the "rescue" of downtown Mexico City: Vision and practice». Latin American Perspectives, 40 (2), 77-94. <https://doi.org/10.1177/0094582X12467762>

BIANCHINI, Franco y GHILARDI, Lia (2007). «Thinking culturally about place». Place Branding and Public Diplomacy, 3 (4), 280-286. $<$ https://doi.org/10.1057/palgrave.pb.6000077>

BianChINI, Franco y PARKInSON, Michael (eds.) (1993). Cultural Policy and Urban Regeneration: The West European Experience. Manchester: Manchester University Press.

BLAKELEY, G. (2005). «Local governance and local democracy: the Barcelona model». Local Government Studies, 31 (2), 149-165.

Bordieu, P. (1997). Capital cultural, escuela y espacio social. México: Siglo XXI Editores.

CARRIÓN, F. (2007). Financiamiento de los centros históricos de América Latina y El Caribe. Quito: Flacso, Lincoln Institute of Land Policy.

Degen, Mónica y García, Marisol (2012). «The transformation of the 'Barcelona model': an analysis of culture, urban regeneration and governance». International Journal of Urban and Regional Research, 36 (5), 1.022-1.038. $<$ https://doi.org/10.1111/j.1468-2427.2012.01152.x>

Delgadillo Polanco, V. M. (2009). «Patrimonio urbano y turismo cultural en la ciudad de México: las chinampas de Xochimilco y el Centro Histórico». Andamios, $6(12), 69-94$. <http://dx.doi.org/10.29092/uacm.v6i12.135>

Díaz FERnÁNDEZ, A. y Ledesma-GómeZ, R. D. (2018). «Un barrio de color: el diseño de un museo al aire libre mediante trencadís en El Nejayote». Panambí. Revista de Investigaciones Artísticas, 6, 55-74.

DINARDI, Cecilia (2012). «Unsettling the role of culture as panacea: The politics of culture-led urban regeneration in Buenos Aires». City, Culture and Society, 6 (2), 9-18. <https://doi.org/10.1016/j.ccs.2015.03.003> 
DUQUe Franco, Isabel (2015). "La cultura como estrategia de transformación y promoción urbana en Bogotá y Medellín». Revista de Geografía Norte Grande, 61, 25-43.

<http://dx.doi.org/10.4067/S0718-34022015000200003>

Duxbury, N.; GarretT-PetTS, W. F. y MacLennan, D. (2015). «Cultural mapping as cultural inquiry: Introduction to an emerging field of practice». En: Cultural mapping as cultural inquiry, 19-60. Nueva York: Routledge.

EIZENBERG, Efrat y COHEN, Nir (2015). «Reconstructing urban image through cultural flagship events: The case of Bat-Yam». Cities, 42, 54-62.

<https://doi.org/10.1016/j.cities.2014.09.003>

Evans, Graeme (2005). "Measure for measure: Evaluating the evidence of culture's contribution to regeneration». Urban Studies, 42 (5-6), 959-983. <https://doi.org/10.1080/00420980500107102>

Evans, G. y FoORD, J. (2008). "Cultural mapping and sustainable communities: planning for the arts revisited». Cultural Trends, 17 (2), 65-96. <https://doi.org/10.1080/09548960802090634>

EVANS, Graeme y SHAW, Phyllida (2004). The contribution of culture to regeneration in the UK: a review of evidence. Londres: DCMS, 4.

Florida, Richard (2005). Cities and the Creative Class. Nueva York y Londres: Routledge.

GARCÍA, Beatriz (2004). «Cultural policy and urban regeneration in Western European cities: lessons from experience, prospects for the future». Local Economy, 19 (4), 312-326. <https://doi.org/10.1080/0269094042000286828>

García, Antonio; FernándeZ, Víctor; CARAVACA, Inmaculada y GonZÁleZ, Gema (2016). "Actividades creativas, transformaciones urbanas y paisajes emergentes. El caso del casco norte de Sevilla». Documents d'Anàlisi Geogràfica, 62 (1), 27-54. <http://dx.doi.org/10.5565/rev/dag.245>

García OrTega, Roberto (2001). «Asentamientos irregulares en Monterrey, 19702000. Divorcio entre planeación y gestión urbana». Frontera Norte, 13 (2), 119-155.

GARZA-RODRÍGUEZ, Fabiola (2015). «Evaluación de indicadores socio-urbanos y estrategias de reforma para el centro de Monterrey». En: VII Seminario Internacional de Investigación en Urbanismo, Barcelona-Montevideo, junio 2015. Departament d'Urbanisme i Ordenació del Territori. Universitat Politècnica de Catalunya.

Geissel, B. (2008). «Do critical citizens foster better governance? A comparative case study». West European Politics, 31 (5), 855-873.

HarveY, David (1989). «From Managerialism to Entrepreneurialism - The Transformation in Urban Governance in Late Capitalism». Geografiska Annaler Series B-Human Geography, 71, 3-17.

- (2005). «El arte de la renta». En: SMITH, N. y HarveY, D. (2005). Capital financiero, propiedad inmobiliaria y cultura. Barcelona: MACBA-Universitat Autònoma de Barcelona.

HAWKES, Jon (2001). The fourth pillar of sustainability: culture's essential role in public planning. Common Ground Publishing Pty Ltd in association with the Cultural Development Network (Vic).

Healey, P. (1996). "The communicative turn in planning theory and its implications for spatial strategy formation». Environment and Planning B: Planning and Design, 23 (2), 217-234. 
- (2003). «Collaborative planning in perspective». Planning Theory, 2 (2), 101-123. <https://doi.org/10.1177/14730952030022002>

Heath, Stacey; Rabinovich, Anna y Barreto, Manuela (2017). «Putting identity into the community: exploring the social dynamics of urban regeneration». European Journal of Social Psychology, 47, 855-866. <http://dx.doi.org/10.1002/ejsp.2296>

JanoschKa, M. y SEQUera, J. (2014). «Procesos de gentrificación y desplazamiento en América Latina, una perspectiva comparativista». En: Michelini, Juan José (ed.). Desafíos metropolitanos. Un diálogo entre Europa y América Latina, 82-104. Madrid: Catarata.

JEANNOTTE, M. S. (2016). «Story-telling about place: Engaging citizens in cultural mapping». City, Culture and Society, 7 (1), 35-41. <https://doi.org/10.1016/j.ccs.2015.07.004>

JONES, Phil y Evans, James (2008). Urban regeneration in the UK: Theory and Practice. Londres: SAGE. <http://dx.doi.org/10.4135/9781473915015>

Jung, Timothy H.; LeE, Jinsik; YaP, Matthew, H. T. y Ineson, Elizabeth M. (2015). "The role of stakeholder collaboration in culture-led urban regeneration: A case study of the Gwangju Project, Korea». Cities, 44, 29-39. <https://doi.org/10.1016/j.cities.2014.12.003>

KANA, Koichi (2012). "An experiment in urban regeneration using culture and art in Senba, Osaka's historic urban center, with a focus on the regeneration of urban space». City, Culture and Society, 3, 151-163. <https://doi.org/10.1016/j.ccs.2012.06.006>

KanAI, Miguel y ORTEGA-AlCÁZAR, Iliana (2009). «The Prospects for Progressive Culture-led Urban Regeneration in Latin America: Cases from Mexico City and Buenos Aires». International Journal of Urban and Regional Research, 33 (2), 483-501. <https://doi.org/10.1111/j.1468-2427.2009.00865.x>

LANDRY, Charles (2000). The creative city: A Toolkit for Urban Innovators. Londres: Earthscan.

- (2003). Imagination and regeneration: Cultural policy and the future of cities. Cultural Policy and Action Department, Directorate General IV, Council of Europe.

LANDRY, Charles y BiAnCHINI, Franco (1995). The creative city. Londres: Demos. Disponible en <http://www.demos.co.uk/files/thecreativecity.pdf>.

LaZAREVIč, Eva Vaništa; KorUŽnjaK, Arch Boris y Devetakovič, Mirjana (2016). "Culture design-led regeneration as a tool used to regenerate deprived areas. Belgrade-The Savamala quarter; reflections on an unplanned cultural zone». Energy and Buildings, 115 (3-10). <http://dx.doi.org/10.1016/j.enbuild.2015.03.015>

LeAl IGA, Carlos y Fitch Osuna, Jesús Manuel (2012). «Políticas urbanas. Tendencia de transformación en el área de influencia de grandes proyectos». Contexto. Revista de la Facultad de Arquitectura de la Universidad Autónoma de Nuevo León, 6 (6).

López SEnABre, D. y Holst, J. (2017). "Centros participativos y comunes urbanos en las políticas culturales de Zaragoza». Clivatge. Estudis i Testimonis sobre el Conflicte i el Canvi Socials, 5, 134-169.

Masso, T. A. di (2007). «Usos retóricos del espacio público: la organización discursiva de un espacio en conflicto". Athenea Digital: Revista de Pensamiento e Investigación Social, 11, 1-22. <https://doi.org/10.5565/rev/athenead/v0n11.335> 
MERcier, G. (2008). «Dimensión cultural de la renovación urbana. Un análisis retórico del urbanismo contemporáneo». Investigación y Desarrollo, 16 (1), 1-15.

MiLeS, Malcom (2005). «Interruptions: Testing the rhetoric of culturally led urban development». Urban Studies, 42 (5-6), 889-911. <https://doi.org/10.1080/00420980500107375>

MirAFTAB, F. (2018). «Insurgencia, planificación y la perspectiva de un urbanismo humano». Territorios, 38, 215-233.

MONTGOMERY, John (2003). «Cultural quarters as mechanisms for urban regeneration. Part 1: Conceptualising cultural quarters». Planning, Practice \& Research, 18 (4), 293-306. <https://doi.org/10.1080/1561426042000215614>

- (2004). "Cultural quarters as mechanisms for urban regeneration. Part 2: A review of four cultural quarters in the UK, Ireland and Australia». Planning, Practice \& Research, 19 (1), 3-31. $<$ https://doi.org/10.1080/0269745042000246559>

Moreno Zúniga, R. y Jurado Montelongo, M. A. (2018). «Expresiones del proceso de gentrificación en el centro de Monterrey». Trayectorias: Revista de Ciencias Sociales de la Universidad Nacional de Nuevo León, 47, 54-76.

Ovalle, Mónica (2007). «El último vestigio del molino El Hércules, en Monterrey, Nuevo León». Boletín de Monumentos Históricos, 10, 56-70.

Parés, M.; Bonet-Martí, J. y Martí-Costa, M. (2012). «Does participation really matter in urban regeneration policies? Exploring governance networks in Catalonia (Spain)». Urban Affairs Review, 48 (2), 238-271.

PETHIA, S. R. (2011). Reconstructing communities: The impact of regeneration on community dynamics and processes. Doctoral dissertation. University of Birmingham).

PRATT, Andy C. (2009). "Urban regeneration: From the arts "feel good" factor to the cultural economy: A case study of Hoxton, London». Urban Studies, 46 (5-6), 1.041-1.061. <https://doi.org/10.1177/0042098009103854>

PRIETO GONZÁlEZ, J. M. (2011). «La consolidación del Monterrey imaginario en el contexto de la globalización: Macroproyectos urbanos». Frontera Norte, 23 (45), 163-191.

- (2016). "Lo humilde en un contexto de "grandeza": Desafíos que enfrenta la regeneración de Barrio Antiguo en Monterrey (Nuevo León)». Contexto. Revista de la Facultad de Arquitectura Universidad Autónoma de Nuevo León, 10 (12), 11-28.

Rius-Ulldemolins, Joaquim y POSSO JiméNEZ, Ladys (2016). "Cultura, transformación urbana y empoderamiento ciudadano frente a la gentrificación. Comparación entre el caso de Getsemaní (Cartagena de Indias) y el Raval (Barcelona)». EURE (Santiago), 42 (126), 97-122. <http://dx.doi.org/10.4067/S0250-71612016000200005>

Roberts, Peter; SyKes, Hugh; Granger, Rachel (eds.) (2016). Urban Regeneration. Londres: SAGE. <http://dx.doi.org/10.4135/9781473921788.n1>

Salgado Gómez, Antonio (2006). «El Barrio Antiguo de Monterrey: ¿Tradición a pesar de todo o transformación a cualquier precio?». En: DELGADO CONTRERAS, Camilo y NARVÁEz Tijerina, Adolfo (coords.). La experiencia de la ciudad y el trabajo como espacios de vida. Tijuana, B. C.: El Colegio de la Frontera Norte.

SHIN, Hyun Bang (2010). "Urban conservation and revalorisation of dilapidated historic quarters: The case of Nanluoguxiang in Beijing». Cities, 27, S43-S54. <https://doi.org/10.1016/j.cities.2010.03.006> 
STERN, M. J. y SEIFERT, S. C. (2010). «Cultural clusters: The implications of cultural assets agglomeration for neighborhood revitalization». Journal of Planning Education and Research, 29 (3), 262-279.

<https://doi.org/10.1177/0739456X09358555>

TALlOn, Andrew (2013). Urban regeneration in the UK. Londres: Routledge.

TRUÑÓ, M. (2008). «Experiencia de Tot Raval y algunas reflexiones sobre el trabajo comunitario". Revista de Educación Social, 7, 11-18.

UN-HABitat (2004). "Cultural strategies for urban development». En: UniTED Nations Human Settlements Programme (2004). The estate of the world cities 2004/2005. Globalization and urban culture, 31-48. Londres: Earthscan. <http://dx.doi.org/10.18356/d1ef8074-en>

YúdicE, George (2008). "Modelos de desarrollo cultural urbano: ¿gentrificación o urbanismo social?». Alteridades, 18 (36), 47-61.

ZaID, Gabriel (2013). Dinero para la cultura. México: Debate.

ZUKIN, Sharon (1987). «Gentrification: culture and capital in the urban core». Annual Review of Sociology, 13 (1), 129-147. <https://doi.org/10.1146/annurev.so.13.080187.001021>

- (1995). The culture of cities. Oxford y Cambridge: Blackwell Publishers. 